\title{
Analysis of an FBMC/OQAM scheme for asynchronous access in wireless communications
}

\author{
Davide Mattera ${ }^{1}$, Mario Tanda ${ }^{1 *}$ and Maurice Bellanger ${ }^{2}$
}

\begin{abstract}
The OFDM/OQAM transceiver belongs to the filter-bank-based multicarrier (FBMC) family and, unlike OFDM schemes, it is particularly able to meet the requirements of the physical layer of cognitive radio networks such as high level of adjacent channel leakage ratio and asynchronous communications. The paper proposes and analyzes a new implementation structure, named frequency spreading, for the OFDM/OQAM transceiver. On flat channels, it is equivalent to the standard one in terms of input-output relations, though more complex. On multipath channels, it offers a crucial advantage in terms of equalization, which is performed in the frequency domain, leading to high performance and no additional delay. With its flexibility and level of performance, the analyzed scheme has the potential to outperform OFDM in the asynchronous access context and in cognitive radio networks.
\end{abstract}

Keywords: OFDM/OQAM; Multicarrier systems; Prototype filter; FBMC; Asynchronous access; Cognitive radio

\section{Introduction}

The cognitive radio transmission context exhibits a number of specific features which make it significantly different from the conventional transmission environment $[1,2]$. First, the available bandwidth is likely to be fragmented, i.e., it is made of non-adjacent spectrum chunks that have to be exploited jointly for high speed data communications. Then, the sections of the spectrum that are not available might be occupied by a primary user and a high level of protection must be provided. Specifically, the transmission system must guarantee a high level of adjacent channel leakage ratio (ACLR) ${ }^{\mathrm{a}}$. Next, the transmission band is likely to be changing on short notice or even without notice. On the exploitation side, the total bandwidth available might be dedicated to a single user requiring high bit-rates or it can be dynamically shared by several users in proportion to their instantaneous capacity needs. If opportunistic operation is contemplated, these users have the freedom to show up and disappear as they wish. In such conditions, a rigid communication procedure, where each user must be aligned before the transmission can start, is inadequate. In fact, asynchronous operation is necessary to reach an acceptable

\footnotetext{
*Correspondence: tanda@unina.it

1 Dipartimento di Ingegneria Elettrica e delle Tecnologie dell'Informazione, Università degli Studi di Napoli Federico II, via Claudio 21, 80125 Napoli, Italy Full list of author information is available at the end of the article
}

level of spectral efficiency. Clearly, to cope with such a context, an appropriate physical layer is required.

The spectrum granularity offered by multicarrier transmission techniques has proven its efficiency for spectrum exploitation, and the most popular technique, orthogonal frequency division multiplexing (OFDM), has been widely used in communications for more than a decade now. However, for the cognitive radio context as described above, it lacks flexibility and it is likely to lead to poor spectral efficiency, even with the introduction of additional processing [3]. Therefore, an enhanced multicarrier technique is needed, as pointed out in [4], where it is shown that a filter-bank-based multicarrier (FBMC) physical layer can meet the ACLR requirements $[2,4]$. In particular, FBMC/OQAM can overcome the limits of OFDM provided that we impose a constraint on the cut-off frequency of the prototype filter, which cannot exceed the sub-channel spacing as pointed out in [5]. High stop-band attenuation filters have been proposed that do not satisfy this constraint, such as isotropic orthogonal transform algorithm (IOTA) [6] and Hermite filters. These filters are associated with single-tap equalizers as mentioned in [7]: such a scenario does not allow the exploitation of the potential advantages of FBMC/OQAM systems for cognitive and, therefore, they cannot compete with CP-OFDM. On the contrary, the high performance equalization objective can be met if the prototype filter (employed in the

\section{是 Springer}

(c) 2015 Mattera et al: licensee Springer. This is an Open Access article distributed under the terms of the Creative Commons Attribution License (http://creativecommons.org/licenses/by/4.0), which permits unrestricted use, distribution, and reproduction in any medium, provided the original work is properly credited. 
FBMC/OQAM scheme) is designed using the frequency sampling technique introduced in [8] and developed in [5]. With this simple approach, the coefficients are derived from a few samples of the filter frequency response, which makes the implementation of the filter bank in the frequency domain practical.

When comparison between such FBMC/OQAM system and OFDM system is considered, it appears that a key advantage of OFDM with cyclic prefix (CP) is the capability to achieve perfect channel equalization, as long as the channel impulse response remains shorter than the guard time provided by the CP. Thus, in order to be accepted, the FBMC/OQAM approach must have a high performance equalization capability, particularly in the asynchronous context, characterized by the fact that the system must compensate simultaneously the timing offset, the frequency offset, and the channel distortion.

In the absence of $\mathrm{CP}$, the equalization capability of the FBMC system rests on the sub-channel equalizers, which cannot be single-tap as in OFDM, but must be multitap to reach similar performance. However, the use of multitap equalizers implies an increase of the receiver latency; this motivates the search for an equalizer structure that does not introduce such a disadvantage. The main contribution of the paper lies in the proposal of a new transceiver structure for FBMC/OQAM systems that is able to provide satisfactory performance without increasing the transceiver delay and accepting the presence of significant timing and frequency offsets among the users that are performing the multiple access, as it is common in a cognitive radio scenario.

The concept has been presented in $[9,10]$, along with preliminary performance results, under the name frequency spreading (FS)-FBMC, but a rigorous analysis of the corresponding scheme is still missing. An objective of the present paper is to provide such an analysis and prove the equivalence of FS-FBMC with the conventional polyphase network (PPN)-FFT scheme in both transmitter and receiver. This equivalence is important because it opens the way to mixed implementations. For example, in uplink transmission, the distant user can be equipped with the conventional IFFT-PPN transmitter, while the high performance but more complex FS-FBMC receiver is implemented at the base station.

Many advances in the applications of FBMC to various scenarios will be able to take advantages from the proposed transceiver structure. In particular, the capability to use multiple antennas at the transmitter and/or at the receiver, which significantly increases bandwidth efficiency, can be easily carried out along the lines introduced in [11-13], which however do not take into account the frequency-despreading structure at each receiver; further works is needed to define the details of the MIMO extension of the proposed structure. Alternative structures are also under consideration for achieving the same goal of operating on multipath channel with a minimum implementation complexity. For example, the fastconvolution structure [14], which is currently under study for its extension on the multipath channel, is superior to the proposed structure in terms of computational complexity. It is equivalent in terms of flexibility (e.g., it shows a similar capability to easily compensate a time-offset in the frequency-domain as suggested in [15]), while the fast-convolution transceiver latency is larger than that achieved by the frequency-despreading system [14].

The organization of the paper is as follows. In Section 2, the FS-FBMC scheme for the transmitter is described and the proof of the equivalence with the standard FBMC, namely the IFFT-PPN cascade, is provided. Section 3 is dedicated to the receiver structures and, again, the proof of the equivalence between FS-FBMC and standard FBMC, namely the cascade of PPN and FFT, is provided; moreover, in Section 3, it is shown that the FSFBMC structure is computationally more complex while in Section 4, it is shown that on a multipath channel, it offers a crucial advantage in terms of equalization, which is performed in the frequency domain, just like OFDM, leading to high performance and no additional delay. In Section 5, the performance of the proposed scheme is illustrated and contrasted with the results obtained for OFDM and standard FBMC when the sub-channel equalizer has a single-tap. In Section 6, the main aspects of FS-FBMC are summarized and the potential impact is discussed.

Notation: $j \triangleq \sqrt{-1}$, superscripts $(\cdot)^{*},(\cdot)^{T}$, and $(\cdot)^{H}$ denote the complex conjugation, the transpose, and the conjugate transpose, respectively, $\Re[\cdot]$ is the real part, log is the base-2 logarithm, $\otimes$ is the linear convolution, $\delta[k]$ is the Kronecker delta, ceil $[x]$ is the smallest integer larger than or equal to $x$, and $\langle\cdot\rangle$ denotes the time average, i.e., $\langle x[n]\rangle \triangleq \lim _{N \rightarrow+\infty} \frac{1}{2 N+1} \sum_{n=-N}^{N} x[n]$ and $\bmod _{M}(\ell) \triangleq$ $\ell-q M$ with $q$ such that $\bmod _{M}(\ell) \in\{0,1, \ldots, M-1\}$. Moreover, we denote with DFT $[\mathbf{x}]$ the vector $\widetilde{\mathbf{x}}$ whose $k$ th component can be written as $\tilde{x}_{k}=\frac{1}{N} \sum_{i=0}^{N-1} x_{i} e^{-j \frac{2 \pi}{N} k i}$ and with IDFT $[\mathbf{x}]$ the vector $\widehat{\mathbf{x}}$ whose $k$ th component can be written as $\hat{x}_{k}=\sum_{i=0}^{N-1} x_{i} e^{\frac{2 \pi}{N} k i}$ where $x_{i}$ is the $i$ th component of the $N \times 1$ input vector $\mathbf{x}$. Finally, lowercase boldface letters denote column vectors, $x$ the componentwise product between two vectors and, finally, $\mathbf{0}$ denotes the null vector.

\section{The transmitter with standard and frequency-spreading structures}

Let us consider an FBMC system using offset QAM modulation, often designated by OFDM-OQAM [16]. We 
assume that the number $M$ of subcarriers be even; the low-pass transmitted signal $s(t)$ can be written as

$$
s(t)=s^{R}(t)+j s^{I}(t-T / 2)
$$

with

$$
\begin{aligned}
& s^{R}(t)=\sum_{n=0}^{N_{b}+N_{s}-1} \sum_{k \in \mathcal{A}} a_{n, k}^{R} e^{j k\left(\frac{2 \pi}{T} t+\frac{\pi}{2}\right)} g(t-n T) \\
& s^{I}(t)=\sum_{n=0}^{N_{b}+N_{s}-1} \sum_{k \in \mathcal{A}} a_{n, k}^{I} e^{j k\left(\frac{2 \pi}{T} t+\frac{\pi}{2}\right)} g(t-n T)
\end{aligned}
$$

where $T$ is the multicarrier symbol interval, $\mathcal{A} \subset$ $\{0,1, \ldots, M-1\}$ is the set of active subcarriers whose size is $M_{u}$, the sequences $a_{n, k}^{R}$ and $a_{n, k}^{I}$ indicate the real and imaginary parts of the complex data symbols transmitted on the $k$ th subcarrier during the $n$th QAM symbol, $N_{b}$ is the number of training symbols, $N_{s}$ is the number of payload symbols, while $g(t)$ is the prototype filter. It is assumed that the data symbols $a_{n, k}^{R}$ and $a_{n, k}^{I}$ are statistically independent with zero-mean and variance $\sigma_{a}^{2}$.

The discrete-time low-pass version $\left.s[i] \triangleq s(t)\right|_{t=i T_{s}}$ of the transmitted signal $\left(T_{s} \triangleq T / M\right.$ is the sampling interval) can be written as

$$
s[i]=s^{R}\left(i T_{s}\right)+j s^{I}\left((i-M / 2) T_{s}\right) .
$$

In the next subsections, we report the derivation of an efficient generation procedure for the signal $s^{R}(t)$. An analogous derivation can be straightforwardly obtained for the signal $s^{I}(t)$. Since the continuous time signal is generated by $\mathrm{D} / \mathrm{A}$ conversion, we consider the generation of its discrete-time samples

$$
s^{R}[i] \triangleq s^{R}\left(i T_{s}\right)=\sum_{n=0}^{N_{b}+N_{s}-1} \sum_{k \in \mathcal{A}} a_{n, k}^{R} e^{j k\left(\frac{2 \pi}{M} i+\frac{\pi}{2}\right)} g[i-n M]
$$

where we have used Equation 2 and the following definition

$$
g[i] \triangleq g\left(i T_{s}\right) .
$$

The generation of the sequence $s^{R}[i]$ is equivalent to the generation of the sequence of $M \times 1$ vectors $\mathbf{d}_{n}^{(R)}$ whose $k$ th component $d_{n, k}^{(R)}$ is equal to $s^{R}[n M+k]$ for $k \in$ $\{0,1, \ldots, M-1\}$. In the following, we consider two implementation structures and their implementation complexities: though the standard implementation structure based on an IFFT over $M$ points exhibits a reduced computational complexity, the frequency-spreading structure based on an IFFT over a larger number of points provides useful insights into the structure of the transmitted signal.

\subsection{Standard transmitter structure}

The standard structure, often named the polyphase network, for the implementation of the OFDM/OQAM transceiver has been first proposed in $[17,18]$. To make clear its comparison with the proposed alternative, we briefly recall its derivation here. The $k$ th component $d_{n, k}^{(R)} \triangleq$ $s^{R}[n M+k]$ of $\mathbf{d}_{n}^{(R)}$ can be written as

$d_{n, k}^{(R)}=\sum_{n^{\prime}=0}^{N_{b}+N_{s}-1} b_{n^{\prime}, k}^{(R)} g\left[k+\left(n-n^{\prime}\right) M\right] \quad k \in\{0,1, \ldots, M-1\}$

where

$b_{n, k}^{(R)} \triangleq \sum_{k^{\prime} \in \mathcal{A}}\left(j^{k^{\prime}} a_{n, k^{\prime}}^{R}\right) e^{j k^{\prime 2} \frac{2 \pi}{M} k} \quad k \in\{0,1, \ldots, M-1\}$

which is the IDFT of the sequence $j^{k} a_{n, k}^{R}$ with respect to the index $k$. If we define the vector $\mathbf{b}_{n}^{(R)}$ as the $M \times 1$ vector whose $k$ th component (for $k \in\{0,1, \ldots, M-1\}$ ) is $b_{n, k}^{(R)}$ in Equation 8, we can compactly write

$$
\mathbf{b}_{n}^{(R)} \triangleq \operatorname{IDFT}\left[\mathbf{w} \times \mathbf{a}_{n}^{(R)}\right]
$$

where IDFT[.] denotes the IDFT operator on the input vector and, for $k \in \mathcal{A}$, the $k$ th component $w_{k}$ of the $M \times 1$-vector $\mathbf{w}$ is

$$
w_{k}=j^{k}
$$

and the $k$ th component of the vector $\mathbf{a}_{n}^{(R)}$ is the symbol $a_{n, k}^{R}$ in Equation 2 while, for $k \notin \mathcal{A}, w_{k}=0$ and the components of $\mathbf{a}_{n}^{(R)}$ are irrelevant. Note that Equation 9 is only defined for $n \in\left\{0,1, \ldots, N_{b}+N_{s}-1\right\}$, but we can straightforwardly extend it to any $n$ provided that we assume that

$$
\mathbf{a}_{n}^{(R)} \equiv \mathbf{b}_{n}^{(R)} \equiv \mathbf{0} \quad n \notin\left\{0,1, \ldots, N_{b}+N_{s}-1\right\} .
$$

We can compactly write Equation 7 as

$$
\mathbf{d}_{n}^{(R)}=\sum_{n^{\prime}=0}^{N_{b}+N_{s}-1} \mathbf{b}_{n^{\prime}}^{(R)} \times \mathbf{g}_{n-n^{\prime}}
$$

where the vector $\mathbf{g}_{n}$ is defined so that its $k$ th component $g_{n, k}$ is

$$
g_{n, k} \triangleq g[k+n M] \quad k \in\{0,1, \ldots, M-1\} .
$$

Therefore, Equation 13 defines the polyphase components of $g[\cdot]$. The prototype filter $g(t)$ satisfies the following property

$$
g(t) \equiv 0 \quad t \notin[0, K T)
$$


where $K$ is the overlap parameter. The property in Equation 14 implies that the vector $\mathbf{g}_{n}$ is nonnull only for $n \in\{0,1, \ldots, K-1\}$. Consequently, Equation 12 can be rewritten as

$$
\mathbf{d}_{n}^{(R)}=\mathbf{g}_{0} \times \mathbf{b}_{n}^{(R)}+\mathbf{g}_{1} \times \mathbf{b}_{n-1}^{(R)}+\ldots+\mathbf{g}_{K-1} \times \mathbf{b}_{n-(K-1)}^{(R)} .
$$

Analogously, the generation of the sequence $s^{(I)}[i]$ in Equation 4 is equivalent to the generation of the sequence of vectors $\mathbf{d}_{n}^{(I)}$ defined as the output of the PPN:

$$
\mathbf{d}_{n}^{(I)}=\mathbf{g}_{0} \times \mathbf{b}_{n}^{(I)}+\mathbf{g}_{1} \times \mathbf{b}_{n-1}^{(I)}+\ldots+\mathbf{g}_{K-1} \times \mathbf{b}_{n-(K-1)}^{(I)}
$$

with

$$
\mathbf{b}_{n}^{(I)} \triangleq \operatorname{IDFT}\left[\mathbf{w} \times \mathbf{a}_{n}^{(I)}\right]
$$

where the $k$ th component $(k \in \mathcal{A})$ of the vector $\mathbf{a}_{n}^{(I)}$ is the symbol $a_{n, k}^{I}$ in Equation 3. Again, we have assumed

$$
\mathbf{a}_{n}^{(I)} \equiv \mathbf{b}_{n}^{(I)} \equiv \mathbf{0} \quad n \notin\left\{0,1, \ldots, N_{b}+N_{s}-1\right\} .
$$

\subsection{Frequency-spreading structure}

In the present subsection, we derive an alternative structure for implementing the OFDM/OQAM transmitter, named the frequency-spreading structure. By using Equations 7 and 8, in Appendix $\mathrm{A}$ it is shown that the $k$ th component $d_{n, k}^{(R)}$ of $\mathbf{d}_{n}^{(R)}$ can be written as:

$$
\begin{aligned}
d_{n, k}^{(R)}= & h_{n, k}+h_{n-1, k+M}+h_{n-2, k+2 M}+h_{n-3, k+3 M} \\
& +\ldots+h_{n-(K-1), k+(K-1) M}
\end{aligned}
$$

where we have defined

$$
h_{m, p} \triangleq g[p] \sum_{k^{\prime} \in \mathcal{A}} c_{m, k^{\prime}}^{(R)} e^{j \frac{2 \pi}{M} p k^{\prime}} \quad p \in\{0,1, \ldots, K M-1\} .
$$

and

$c_{n^{\prime}, k^{\prime}}^{(R)} \triangleq\left\{\begin{array}{cc}j^{k^{\prime}} a_{n^{\prime}, k^{\prime}}^{R} & n^{\prime} \in\left\{0,1, \ldots, N_{b}+N_{s}-1\right\} \text { and } k^{\prime} \in \mathcal{A} \\ 0 & n^{\prime} \notin\left\{0,1, \ldots, N_{b}+N_{s}-1\right\} \text { and } \forall k^{\prime}\end{array}\right.$

Equation 19, which has to be applied for $k \in\{0,1, \ldots, M-1\}$, is similar to that present in the classic fast-convolution procedure, usually named overlap-and-add structure; here, it operates on the sequence of $K M \times 1$ vectors $\mathbf{h}_{n}$, defined as follows:

$$
\mathbf{h}_{m} \triangleq\left[h_{m, 0} h_{m, 1} \ldots h_{m, K M-1}\right]^{T}
$$

More specifically, let us first define the vectors $\mathbf{h}_{m}^{(i)}$ such that

$$
\begin{aligned}
\mathbf{h}_{m}^{(i)} \triangleq & {\left[h_{m, i M} h_{m, i M+1} h_{m, i M+2} \ldots h_{m, i M+M-1}\right]^{T} } \\
& i \in\{0,1, \ldots, K-1\} .
\end{aligned}
$$

From Equations 22 and 23, it follows that

$$
\mathbf{h}_{m}=\left[\begin{array}{llll}
\mathbf{h}_{m}^{(0) T} \mathbf{h}_{m}^{(1) T} & \ldots & \mathbf{h}_{m}^{(K-1) T}
\end{array}\right]^{T} .
$$

Using Equations 23 and 24, Equation 19 can be re-written as

$$
\mathbf{d}_{n}^{(R)}=\mathbf{h}_{n}^{(0)}+\mathbf{h}_{n-1}^{(1)}+\mathbf{h}_{n-2}^{(2)}+\cdots+\mathbf{h}_{n-(K-1)}^{(K-1)} .
$$

We have now to study the structure of the vector $\mathbf{h}_{n}$ in Equation 24 for a general time-step $n$ in order to simplify its generation. From Equation 20, it follows that its $k$ th component $h_{n, k}$ can be written as

$$
\begin{aligned}
h_{n, k} & \triangleq g[k] \sum_{k^{\prime} \in \mathcal{A}} c_{n, k^{\prime}}^{(R)} e^{j \frac{2 \pi}{M} k k^{\prime}} \quad k \in\{0,1, \ldots, K M-1\} \\
& =\left\{\sum_{k^{\prime}=-(K-1)}^{K-1} G_{k^{\prime}} e^{j \frac{2 \pi}{K M} k k^{\prime}}\right\}\left\{\sum_{k^{\prime} \in \mathcal{A}} c_{n, k^{\prime}}^{(R)} e^{j \frac{2 \pi}{K M} K k k^{\prime}}\right\} \\
& =\left\{\sum_{k^{\prime}=-(K-1)}^{K-1} G_{k^{\prime}} e^{j \frac{2 \pi}{K M} k k^{\prime}}\right\}\left\{\sum_{m=0}^{K M-1} c_{n, m}^{(K R)} e^{j \frac{2 \pi}{K M} k m}\right\}(26)
\end{aligned}
$$

where we have assumed that the prototype filter has been designed according to a frequency-sampling approach so that its Fourier transform

$$
G(F) \triangleq \sum_{i=0}^{K M-1} g[i] e^{-j 2 \pi F i}
$$

satisfies the property

$$
G(F)_{\left.\right|_{F=\frac{k}{K M}}}=0 \quad k \in\{K, K+1, \ldots, K M-K\} .
$$

The Fourier coefficients in Equation 26 are given by

$$
G_{k} \triangleq \frac{1}{K M} G(F)_{\left.\right|_{F=\frac{k}{K M}}} \quad \forall k .
$$

Note that the $2 K-1$ nonnull values $\left\{G_{k}\right\}_{k=-(K-1)}^{K-1}$ are the free parameters of the prototype filter when it is designed according to the frequency-sampling procedure used in [5].

Furthermore, in Equation 26, we have also introduced the following definition of the $K$-times upsampled version of $c_{n, k}^{(R)}$ in Equation 21:

$$
c_{n, m}^{(K R)} \triangleq\left\{\begin{array}{cc}
c_{n, k}^{(R)} & m=k K \\
0 & \text { otherwise }
\end{array} .\right.
$$

From Equation 26, it follows that

$$
\mathbf{h}_{n}=\operatorname{IDFT}[\mathbf{G}] \times \operatorname{IDFT}\left[\mathbf{c}_{n}\right]=\operatorname{IDFT}\left[\mathbf{z}_{n}\right]
$$


where the vectors $\mathbf{G}$ and $\mathbf{c}_{n}$ in Equation 31 are defined as follows:

$$
\begin{aligned}
& \mathbf{G} \triangleq\left[\begin{array}{lllll}
G_{0} & G_{1} & G_{2} & \ldots & G_{K M-1}
\end{array}\right]^{T}
\end{aligned}
$$

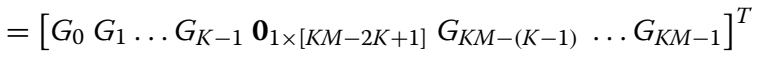

$$
\begin{aligned}
\mathbf{c}_{n} \triangleq & {\left[c_{n, 0}^{(R)} \mathbf{0}_{1 \times(K-1)} c_{n, 1}^{(R)} \mathbf{0}_{1 \times(K-1)} c_{n, 2}^{(R)} \mathbf{0}_{1 \times(K-1)} \cdots\right.} \\
& \left.c_{n, M-1}^{(R)} \mathbf{0}_{1 \times(K-1)}\right]^{T}
\end{aligned}
$$

and the vector $\mathbf{z}_{n}$ in Equation 31 for the well-known property of the DFT and IDFT operators is the circular convolution of the two $K M \times 1$ vectors $\mathbf{G}$ and $\mathbf{c}_{n}$. We have used the property in Equation 28 to write Equation 33. In Appendix B, it is shown that the components of the vector $\mathbf{z}_{n}$ in Equation 31 can be written as

$$
z_{n, p K+k}=j^{p}\left[a_{n, p}^{R} G_{k}+j a_{n, \bmod _{M}(p+1)}^{R} G_{k-K}(1-\delta[k])\right]
$$

where $p \in\{0,1, \ldots, M\}$ and $k \in\{0,1, \ldots, K-1\}$.

Therefore, the frequency-spreading structure requires:

1. to use the input symbols $a_{n, k}^{R}$ to calculate (for $\ell \in\{0,1, \ldots, K M-1\})$ the components $z_{n, \ell}$ of $\mathbf{z}_{n}$ according to Equation 35 . Note that the symbol $a_{n, p}^{R}$ is spread over $2 K-1$ components of the vector $\mathbf{z}_{n}$ and for this reason, the structure is named FS-FBMC; in fact, each component of $\mathbf{z}_{n}$ is dependent on two adjacent symbols and each symbol $a_{n, p}$, according to Equation 35, not only determines the component $p K$ of the frequency-domain vector $\mathbf{z}_{n}$ but also spreads its effect, weighted by the frequency response of the prototype filter, on the different components of the same vector ranging from $p K-(K-1)$ up to $p K+K-1$

2. to determine $\mathbf{h}_{n}$ starting from $\mathbf{z}_{n}$ by performing the IDFT over KM points in the right-hand side of Equation 31;

3. to evaluate $\mathbf{d}_{n}^{(R)}$ by the overlap-and-add processing defined in Equations 24 and 25.

\subsection{Complexity comparison of the two structures}

The standard transmitter structure requires to calculate (a) the IFFT over $M$ samples according to the definition in Equation 9 then to calculate the vector $\mathbf{d}_{n}^{(R)}$ according to the PPN (Equation 15). The frequency-spreading structure requirements have been just summarized.

In a structure with a single processor, the complexity comparison is equivalent to the count of the number of flops required by the two structures. The number of complex flops for calculating the IFFT over $M$ samples can be written as $1.5 M \log (M)$ while the number of real flops can be written ${ }^{\mathrm{b}}$ as $4 M \log (M)-6 M+8$ as in the split-radix.

The number of complex multiplications for calculating IFFT over $M$ samples can be written as $0.5 M \times(\log M-1)$ while the number of real multiplications can be written as $M \log (M)-3 M+4$ [19] by removing most of the trivial operations and using three real multiplications per complex multiplication.

To calculate the vector $\mathbf{d}_{n}^{(R)}$ according to the PPN (Equation 15), the following number of real-valued flops are necessary: $2 M$ real-valued multiplications for each of the $K$ terms and $M$ complex-valued additions for each of the $K-1$ couples of vectors to be summed.

With the frequency-spreading structure, we need $2 K M-M$ real-valued multiplications for calculating $\mathbf{z}_{n}$ according to Equation 35 while the IFFT for calculating $\mathbf{h}_{n}$ by using Equation 31 requires $4 K M \log (K M)-6 K M+8$ real-valued flops. Finally, $(K-1) M$ complex-valued additions are needed by the overlap-and-sum structure in Equation 25.

Therefore, for generating each vector $\mathbf{d}_{n}^{(R)}$, with the standard structure, we need $C_{f}^{(S T)}$ real-valued flops or $C_{m}^{(S T)}$ real-valued multiplications where

$$
\begin{aligned}
C_{f}^{(S T)} & =\underbrace{4 M \log (M)-6 M+8}_{(9)}+\underbrace{2 M K+2 M(K-1)}_{(15)} \\
& =8+M[4 \log (M)+4 K-8] \\
C_{m}^{(S T)} & =\underbrace{M \log (M)-3 M+4}_{(9)}+\underbrace{2 M K}_{(15)}=M[\log (M)+2 K-3]+4
\end{aligned}
$$

while with the frequency-spreading structure, we need $C_{f}^{(F T)}$ real-valued flops or $C_{m}^{(F T)}$ real-valued multiplications where

$$
\begin{aligned}
C_{f}^{(F T)} & =\underbrace{2 K M-M}_{(35)}+\underbrace{4 K M \log (K M)-6 K M+8}_{(31)}+\underbrace{2(K-1) M}_{(25)} \\
& =8+M\{4 K[\log (K M)-0.5]-3\} \\
C_{m}^{(F T)} & =\underbrace{2 K M-M}_{(35)}+\underbrace{K M \log (K M)-3 K M+4}_{(31)} \\
& =M\{K[\log (K)+\log (M)-1]-1\}+4 .
\end{aligned}
$$

Note that

$$
\begin{aligned}
C_{f}^{(F T)} & \simeq M K[4 \log (M)+4 \log (K)-2] \\
& \simeq M K[4 \log (M)+4 K-8]=K C_{f}^{(S T)} .
\end{aligned}
$$

The first approximation is obvious while the second approximation holds provided that $M$ is sufficiently large and $K$ sufficiently small (e.g., for $K=4$ and $M=1024$, we 
have a normalized approximation error of 6.1\%). Moreover, $C_{m}^{(S T)} / C_{m}^{(F T)}$ is around $58 \%$ for $K=2,35 \%$ for $K=4$, and $24 \%$ for $K=8$, mainly independently of $M \in\{512,1024,2048,4096\}$. Therefore, the frequencyspreading structure is about $K$ times more complex when implemented using a structure with a single processor.

\section{The receiver for standard and frequency-spreading structures}

According to Equations 4 and 5, assuming perfect synchronization on the flat channel (i.e., the channel with unit response in the frequency domain) and neglecting the presence of the noise, the received signal $r[i]$ can be written as follows

$$
\begin{aligned}
r[i]= & \alpha \sum_{n=0}^{N_{b}+N_{s}-1} \sum_{k \in \mathcal{A}}\left\{a_{n, k}^{R} e^{j k\left(\frac{2 \pi}{M} i+\frac{\pi}{2}\right)} g[i-n M]\right. \\
& \left.+j a_{n, k}^{I}(-1)^{k} e^{j k\left(\frac{2 \pi}{M} i+\frac{\pi}{2}\right)} g[i-n M-M / 2]\right\} \\
= & \alpha a_{n, k}^{R} e^{j k\left(\frac{2 \pi}{M} i+\frac{\pi}{2}\right)} g[i-n M]+o^{(R)}[i] \\
= & j \alpha a_{n, k}^{I}(-1)^{k} e^{j k\left(\frac{2 \pi}{M} i+\frac{\pi}{2}\right)} g[i-n M-M / 2]+o^{(I)}[i]
\end{aligned}
$$

where $0<\alpha<1$ denotes the channel gain and $o^{(R)}[i]$ denotes terms present in the transmitted signal that do not depend on $a_{n, k}^{R}$ while $o^{(I)}[i]$ denotes terms present in the transmitted signal that do not depend on $a_{n, k}^{I}$, with $n \in\left\{0,1, \ldots, N_{b}+N_{s}\right\}$ and $k \in \mathcal{A}$. We consider at the receiver the decision variables:

$$
\begin{aligned}
& \hat{a}_{n, k}^{R} \triangleq \Re\left\{D_{n, k}^{(R)}\right\} \quad D_{n, k}^{(R)} \triangleq \frac{1}{A} \sum_{i=-\infty}^{+\infty} r[i] e^{-j k\left(\frac{2 \pi}{M} i+\frac{\pi}{2}\right)} g[i-n M \\
& \hat{a}_{n, k}^{I} \triangleq \Re\left\{D_{n, k}^{(I)}\right\} \quad D_{n, k}^{(I)} \triangleq \frac{-j}{A} \sum_{i=-\infty}^{+\infty} r[i](-1)^{k} e^{-j k\left(\frac{2 \pi}{M} i+\frac{\pi}{2}\right)} \\
& \times g[i-n M-M / 2]
\end{aligned}
$$

where $A$ is a proper constant amplitude, defined so that $\hat{a}_{n, k}^{(R)}=a_{n, k}^{R}$ when $o^{(R)}[i]=0$ and $\hat{a}_{n, k}^{(I)}=a_{n, k}^{I}$ when $o^{(I)}[i]=$ 0 . Consequently,

$$
A=\alpha \mathcal{E}_{g}
$$

with $\mathcal{E}_{g} \triangleq \sum_{i=0}^{K M-1} g^{2}[i]$. Equation 41 is motivated by a proper design of the prototype filter $g[\cdot]$ that guarantees a negligible projection of the interference terms $o^{(R)}[i]$ on the matched filter, as usual in FBMC receiver $[16,20,21]$ :

$$
\Re\left\{\sum_{i=-\infty}^{+\infty} o^{(R)}[i] e^{-j k\left(\frac{2 \pi}{M} i+\frac{\pi}{2}\right)} g[i-n M]\right\} \simeq 0 .
$$

In fact, according to Equation 39, $o^{(R)}[i]$ denotes the additive signals present in the received signal $r[i]$ that do not depend on the useful symbol $a_{n, k}^{R}$; the condition in Equation 44 therefore implies that such additive signals do not interfere with the useful signal when the matchedfilter projection (designed according to the useful term) is performed (i.e., the result of the matched-filter projection is independent of the interfering signals); on the other hand, the matched-filter projection is optimum (in the maximum-likelihood sense) when the interference signals are not present and only the noisy version of the useful term is taken into account. Consequently, the decision variable in Equation 41 operating on the flat channel without synchronization error is optimum (in the maximumlikelihood sense) for estimating statistically independent information symbols; analogously, the same optimality holds for the decision variable in Equation 42. Obviously, the receiver implemented according to Equation 41 has to be modified in order to operate on a multipath channel. However, before discussing such modifications, we first need to describe the two structures implementing Equation 41. Consequently, we recall the standard structure for implementing Equation 41 in Subsection 3.1 and we introduce an alternative structure in Subsection 3.2; moreover, we compare their complexities in Subsection 3.3.

\subsection{The standard receiver structure}

In the present subsection, we briefly recall the derivation of the standard receiver structure. From Equations 41 and 14 , it follows that

$$
\begin{aligned}
D_{n, k}^{(R)}= & \frac{1}{A} j^{-k}\left\{\sum_{i=0}^{M-1} r[n M+i] e^{-j k \frac{2 \pi}{M} i} g[i]\right. \\
& +\sum_{i=0}^{M-1} r[(n+1) M+i] e^{-j k \frac{2 \pi}{M} i} g[M+i] \\
& +\sum_{i=0}^{M-1} r[(n+2) M+i] e^{-j k \frac{2 \pi}{M} i} g[2 M+i]+\ldots \\
& \left.+\sum_{i=0}^{M-1} r[(n+K-1) M+i] e^{-j k \frac{2 \pi}{M} i} g[(K-1) M+i]\right\} .
\end{aligned}
$$

Let us introduce the vector $\mathbf{r}_{n}$ whose $i$ th component $r_{n, i}$ $(i \in\{0,1, \ldots, M-1\})$ is defined as follows

$$
r_{n, i} \triangleq r[n M+i]
$$

By using also Equation 13, let us consider the vector

$$
\mathbf{r}_{n+\ell}^{(g, n)} \triangleq \mathbf{r}_{n+\ell} \times \mathbf{g}_{\ell} \quad \ell \in\{0,1, \ldots, K-1\}
$$


and let us denote with $r_{n+\ell, i}^{(g, n)}$ its $i$ th component for $i \in$ $\{0,1, \ldots, M-1\}$. Using such definitions, from Equation 45, it follows that

$$
\begin{aligned}
A j^{k} D_{n, k}^{(R)}= & \sum_{q=0}^{M-1} r_{n, q}^{(g, n)} e^{-j k \frac{2 \pi}{M} q}+\sum_{q=0}^{M-1} r_{n+1, q}^{(g, n)} e^{-j k \frac{2 \pi}{M} q} \\
& +\sum_{q=0}^{M-1} r_{n+2, q}^{(g, n)} e^{-j k \frac{2 \pi}{M} q}+\ldots+\sum_{q=0}^{M-1} r_{n+(K-1), q}^{(g, n)} e^{-j k \frac{2 \pi}{M} q}
\end{aligned}
$$

Defining the vector $\mathbf{D}_{n}^{(R)} \triangleq\left[D_{n, 0}^{(R)} D_{n, 1}^{(R)} \ldots D_{n, M-1}^{(R)}\right]^{T}$ containing the decision variables on the vector $\mathbf{a}_{n}^{(R)}$, it follows from Equation 48 that

$$
\begin{aligned}
A \mathbf{D}_{n}^{(R)} \times \mathbf{w}= & M \operatorname{DFT}\left[\mathbf{r}_{n}^{(g, n)}\right]+M \operatorname{DFT}\left[\mathbf{r}_{n+1}^{(g, n)}\right]+\ldots \\
& +M \operatorname{DFT}\left[\mathbf{r}_{n+(K-1)}^{(g, n)}\right] \\
= & M \sum_{\ell=0}^{K-1} \operatorname{DFT}\left[\mathbf{r}_{n+\ell} \times \mathbf{g}_{\ell}\right]
\end{aligned}
$$

or, equivalently,

$$
\mathbf{D}_{n}^{(R)}=M \frac{\mathbf{w}^{*}}{A} \times \operatorname{DFT}\left[\sum_{\ell=0}^{K-1} \mathbf{r}_{n+\ell} \times \mathbf{g}_{\ell}\right] .
$$

Analogously, in Appendix $\mathrm{C}$, it is shown that

$$
\mathbf{D}_{n}^{(I)}=M \frac{\mathbf{w}^{*}}{j A} \times \operatorname{DFT}\left[\sum_{\ell=0}^{K-1} \mathbf{r}_{n+\ell}^{(I)} \times \mathbf{g}_{\ell}\right]
$$

where the vector $\mathbf{D}_{n}^{(I)}$ contains the decision variables on the vector $\mathbf{a}_{n}^{(I)}$ :

$$
\mathbf{D}_{n}^{(I)} \triangleq\left[D_{n, 0}^{(I)} D_{n, 1}^{(I)} \ldots D_{n, M-1}^{(I)}\right]^{T}
$$

while the $i$ th component $r_{n, i}^{(I)}(i \in\{0,1, \ldots, M-1\})$ of the vector $\mathbf{r}_{n}^{(I)}$ is defined as follows

$$
r_{n, i}^{(I)} \triangleq r[n M+M / 2+i] \text {. }
$$

\subsection{The frequency-despreading receiver structure}

In the present subsection, we derive the receiver counterpart of the frequency-spreading transmitter described in Subsection 2.2. From Equation 41, it follows that for $k \in\{0,1, \ldots, M-1\}$

$$
\begin{aligned}
D_{n, k}^{(R)} & =\frac{1}{A} j^{-k} \sum_{i=0}^{K M-1} r[n M+i] e^{-j k \frac{2 \pi}{M} i}\left\{\sum_{k^{\prime}=-(K-1)}^{K-1} G_{k^{\prime}} e^{j \frac{2 \pi}{K M} i k^{\prime}}\right\} \\
& =\frac{1}{A} j^{-k} \sum_{k^{\prime}=-(K-1)}^{K-1} G_{k^{\prime}}^{*} \sum_{i=0}^{K M-1} r[n M+i] e^{-j \frac{2 \pi}{K M} i\left(k K+k^{\prime}\right)}
\end{aligned}
$$

where we have taken into account the properties of the prototype filter (see Equations 14, 28, and 29) and the fact that it is real and, consequently, $G_{-k}=G_{k}^{*}$.

Let us introduce the $K M \times 1$ vector $\mathbf{r}_{n}^{(K)}$ whose $k$ th component $r_{n, k}^{(K)}$ is defined as

$$
r_{n, k}^{(K)} \triangleq r[n M+k] \quad k \in\{0,1, \ldots, K M-1\}
$$

and the vector $\mathbf{R}_{n}$, whose $k$ th component is denoted with $R_{n, k}$, defined as the DFT over KM samples of the vector $\mathbf{r}_{n}^{(K)}$ :

$$
\mathbf{R}_{n} \triangleq \operatorname{DFT}\left[\mathbf{r}_{n}^{(K)}\right]
$$

Using such definitions, Equation 54 can be re-written as

$$
D_{n, k}^{(R)}=\frac{1}{A} j^{-k} K M \sum_{k^{\prime}=-(K-1)}^{K-1} G_{k^{\prime}}^{*} R_{n, \bmod _{K M}\left(k K+k^{\prime}\right)} k \in\{0,1, \ldots, M-1\} .
$$

Let us define the $(2 K-1) \times 1$ vector $\mathbf{R}_{n, k}^{(F S)}(k \in\{0,1, \ldots$, $M-1\})$

$$
\begin{aligned}
\mathbf{R}_{n, k}^{(F S) \triangleq} & {\left[R_{n, \bmod _{K M}(k K-(K-1))} \ldots R_{n, \bmod _{K M}(k K)} \ldots\right.} \\
& R_{\left.n, \bmod _{K M}(k K+(K-1))\right]^{T}}
\end{aligned}
$$

and the vector

$$
\mathbf{G}^{(K)} \triangleq\left[G_{-(K-1)} \ldots G_{-1} G_{0} G_{1} \ldots G_{K-1}\right]^{T} .
$$

Taking into account Equations 58 and 59, Equation 57 becomes

$$
D_{n, k}^{(R)}=\frac{K M}{A} j^{-k}\left[\mathbf{G}^{(K)^{H}} \mathbf{R}_{n, k}^{(F S)}\right] .
$$

Analogously, in Appendix D, it is shown that the decision variable on $a_{n, k}^{(I)}$ can be written as

$$
D_{n, k}^{(I)}=\frac{K M}{A} j^{-k-1}\left[\mathbf{G}^{(K)^{H}} \mathbf{R}_{n, k}^{(I, F S)}\right]
$$

where we define the $(2 K-1) \times 1$ vector $\mathbf{R}_{n, k}^{(I, F S)}(k \in$ $\{0,1, \ldots, M-1\})$ as follows

$$
\begin{aligned}
\mathbf{R}_{n, k}^{(I, F S) \triangleq} & {\left[R_{n, \bmod _{K M}(k K-(K-1))}^{(I)} \ldots R_{n, \bmod _{K M}(k K)}^{(I)} \cdots\right.} \\
& \left.R_{n, \bmod _{K M}(k K+(K-1))}^{(I)}\right]^{T} .
\end{aligned}
$$

The vector $\mathbf{R}_{n}^{(I)}$

$$
\mathbf{R}_{n}^{(I)} \triangleq \operatorname{DFT}\left[\mathbf{r}_{n}^{(I, K)}\right]
$$


whose $k$ th component is denoted with $R_{n, k}^{(I)}$ and used in Equation 62 is defined as the DFT over $K M$ samples of the vector $\mathbf{r}_{n}^{(I, K)}$ whose $k$ th component $r_{n, k}^{(I, K)}$ is defined as

$r_{n, k}^{(I, K)} \triangleq r[n M+M / 2+k] \quad k \in\{0,1, \ldots, K M-1\}$.

Therefore, Equations 60 and 61 define the following receiver structure, illustrated in Figure 1:

1. Collect the samples of the received signal $r[\cdot]$ to form the $K M \times 1$ vector $r_{n, k}^{(K)}$ in Equation 55 (and half period later the vector $r_{n, k}^{(I, K)}$ in Equation 64);

2. Calculate the vector $\mathbf{R}_{n}$ in Equation 56 by FFT over $K M$ points (and half period later the vector $\mathbf{R}_{n}^{(I)}$ in Equation 63);

3. Perform $M$ different projections of the vector $\mathbf{R}_{n}$ according to Equations 60 and 58 in order to obtain each decision variable $D_{n, k}^{(R)}$ for $k \in\{0,1, \ldots, M-1\}$ (and half period later according to Equations 61 and 62 to obtain $\left.D_{n, k}^{(I)}\right)$. Thus, to obtain the datum $a_{n, k}^{(R)}$ that in the transmitter has been spread over $2 K-1$ components of the vector $\mathbf{z}_{n}$ (see the first point of the sentence after Equation 35), the same components of the vector $\mathbf{R}_{n}$ are exploited by using as weights the (conjugate) Fourier coefficients $G_{k^{\prime}}^{*}$ with $k^{\prime} \in\{-(K-1), \ldots, K-1\}$. For this reason, such a structure is called frequency-despreading receiver: in fact, it collects all the components of the frequency-domain vector $\mathbf{R}_{n}$ dependent on the useful symbol $a_{n, k}^{R}$, due to the spreading performed at the transmitter, and weights them according to the frequency response of the prototype filter, achieving the despreading of the useful symbol.

The importance of the proposed structure is not limited by the assumption in Equation 28 because, when it is necessary to introduce a possible mismatch (i.e., to use at the transmitter a prototype filter that does not satisfy Equation 28), it can be managed with very marginal performance loss (i.e., the frequency despreading receiver can be still employed at the receiver, with its advantages considered in the paper and without appreciable disadvantages due to the presence of a mismatch).

\subsection{Complexity comparison of the two structures}

The standard receiver structure (see Equation 50) requires to calculate (a) $M K$ multiplications, (b) $K-1$ additions of $M \times 1$ vectors, and (c) an FFT over $M$ samples, which is the same requirement of the standard transmitter structure. Therefore, the overall number of real-valued flops $C_{f}^{S R}$ and the overall number of real-valued multiplications $C_{m}^{S R}$ are equal to their transmitter counterpart $C_{f}^{S T}$ and $C_{m}^{S T}$ in Equation 36. The frequency-despreading structure

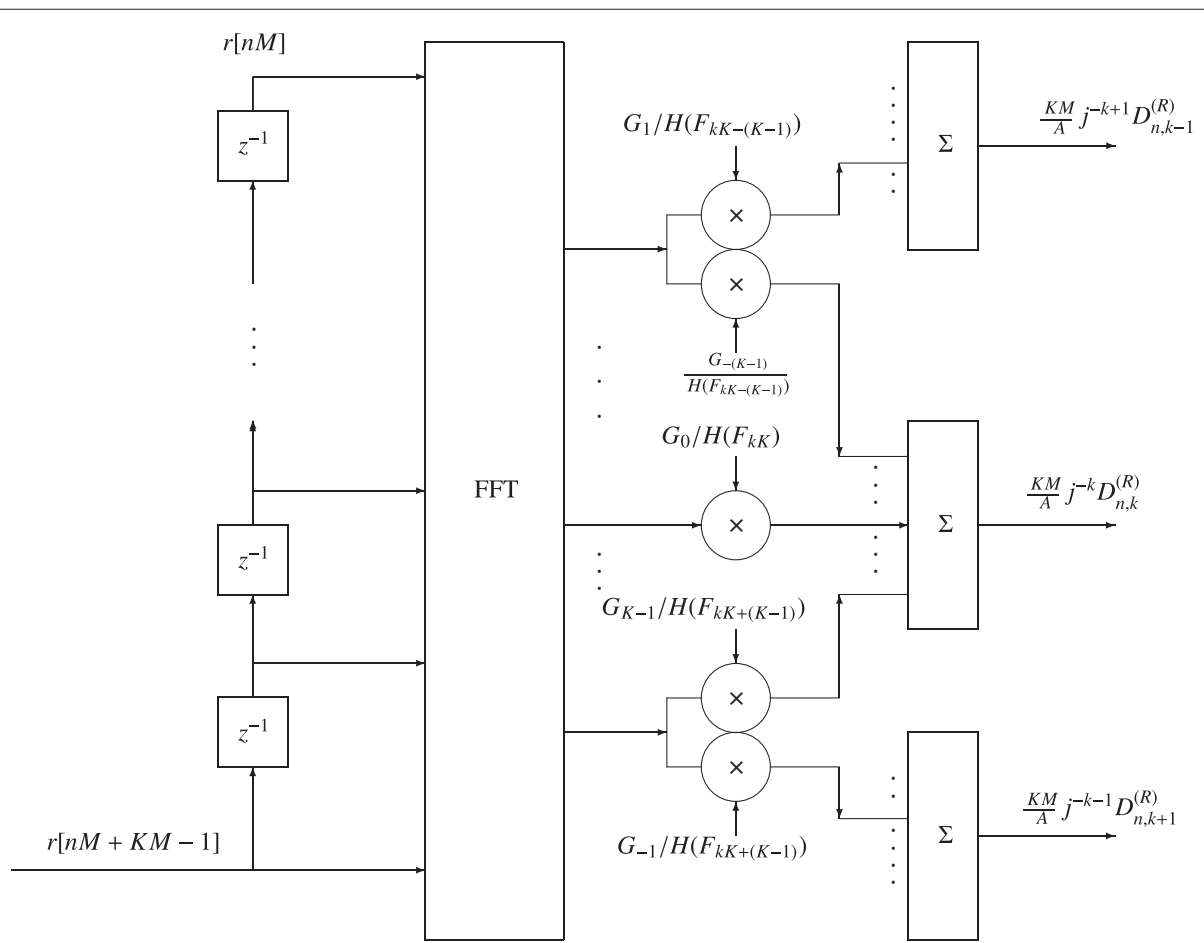

Figure 1 Frequency despreading. The frequency despreading structure at the receiver side. On a flat channel $H_{m}=1$ for any $m$ while on a multipath channel, the values of $H_{m}$ depends on the channel frequency response and are used to equalize the channel distortions. 
is described in Equations 60, 58, and 56. With reference to such a structure, the overall number of real-valued flops $C_{f}^{F R}$ and the overall number of real-valued multiplications $C_{m}^{F R}$ can be written as

$$
\begin{aligned}
C_{f}^{F R}= & \underbrace{4 K M \log (K M)-6 K M+8}_{\text {FFT in Equation } 56} \\
& +[\underbrace{2(2 K-1)}_{\text {multiplications in Equation } 60 \text { additions in Equation } 60}+\underbrace{M} \\
& \times \underbrace{2(2 K-2)}_{k \in\{0,1 \ldots, M-1\} \text { in Equation } 60} \\
= & 8+M[4 K \log (K M)+2 K-6]
\end{aligned}
$$

$$
\begin{aligned}
C_{m}^{F R}= & \underbrace{K M \log (K M)-3 K M+4}_{\text {FFT in Equation } 56}+ \\
& \underbrace{M}_{k \in\{0,1 \ldots, M-1\} \text { in Equation } 60} \\
= & 4+M[K \log (K M)+K-2] .
\end{aligned}
$$

Note that

$$
\begin{aligned}
C_{f}^{F R} & =M K[4 \log (M)+4 \log (K)+2]+8-6 M \\
& \simeq M K[4 \log (M)+4 K-8] \simeq K C_{f}^{S R}
\end{aligned}
$$

where the first and the second approximations are obvious while the third one holds provided that $M$ is sufficiently large and $K$ is sufficiently small (e.g, for $M=1024$ and $K=4,4 \log (M)+4 \log (K)+2=50$ while $4 \log (M)+$ $4 K-8=48)$. Therefore, under the same assumptions used at the transmitter side, we can obtain the following approximation: $C_{f}^{F R}=K C_{f}^{S R}$; moreover, $C_{m}^{S R} / C_{m}^{F R}$ is about $50 \%$ for $K=2$, $30 \%$ for $K=4$, and $21 \%$ for $K=8$, independently of $M \in\{512,1024,2048,4096\}$. Thus, also for the receiver case, the frequency-spreading structure has a computational complexity about $K$ times larger.

The complexities of the different considered structures are summarized in Table 1 . Note that the analysis shows that the complexity increase is due to the fact that the frequency-spreading structure requires to evaluate the DFT over $K M$ points instead of the DFT over $M$ points required by the standard structure. Since many solutions exist for implementing FFT processing, the result of the comparison depends on the particular solution for its implementation; however, a $K$-fold increase of the implementation costs can be considered an upper-bound; not always, however, the installed processing power can be optimized to the effective needs and, consequently, the increase of the actual costs (needed to perform the FFT over a larger number of points) can be much smaller depending on the effective implementation details.

\section{Adapting the frequency-despreading structure to the multipath channel}

In the present section, we first define the adaptation of the frequency-despreading structure to the multipath channel, then we recall a standard approach to adapt the standard structure to the multipath channel and we finally compare their performance.

\subsection{Frequency-despreading structure operating on multipath channel}

When the multipath nature of the channel is taken into account, the received signal $r[i]$ can be written as

$$
r[i]=\sum_{\ell=0}^{L_{h}} h[\ell] s[i-\ell]+\eta[i]
$$

where the complex-valued sequence $h[i]$ of length $L_{h}+1$ models the multipath channel.

In Equation 67, the noise term $\eta[i]$ is assumed to be the $i$ th sample of the low-pass equivalent of a white Gaussian random process with null average and power spectral density equal to $N_{0} / 2$ in the signal bandwidth; the analog low-pass equivalent has null average and power spectral density equal to $2 N_{0}$. The low-pass equivalent is sampled with sampling period $T_{s}$; assuming an ideal antialiasing filter with bandwidth equal to $1 /\left(2 T_{s}\right)$, the random variable $\eta[i]$ ideally sampled at the filter output has null average and variance $\operatorname{VAR}[\eta[i]]=2 N_{0} / T_{s}$. Moreover, the sequence $\eta[i]$ is zero-mean, white, Gaussian and circularly symmetric; thus,

$E\left[\eta[n] \eta^{*}[n-m]\right]=\frac{2 N_{0}}{T_{s}} \delta[m] \quad E[\eta[n] \eta[n-m]] \equiv 0$.

Table 1 Structure complexity

\begin{tabular}{lcc}
\hline Structure & Number of flops & Number of multiplications \\
\hline Standard transmitter & $8+M[4 \log (M)+4 K-8]$ & $M[\log (M)+2 K-3]+4$ \\
\hline Standard receiver & $8+M[4 \log (M)+4 K-8]$ & $M[\log (M)+2 K-3]+4$ \\
\hline FS transmitter & $8+M\{4 K[\log (K M)-0.5]-3\}$ & $M\{K[\log (K)+\log (M)-1]-1\}+4$ \\
\hline FS receiver & $8+M[4 K \log (K M)+2 K-6]$ & $4+M[K \log (K M)+K-2]$ \\
\hline
\end{tabular}

The number of flops and the number of multiplications of the two transceiver structures for each half multicarrier symbol period. 
On a multipath channel, an equalization stage has to be included in the structures already described in Section 3. In particular, when the frequency-despreading receiver (see Equation 57) is considered, the following modified structure is considered

$D_{n, k}^{(R)}=\frac{1}{A} j^{-k} K M \sum_{k^{\prime}=-(K-1)}^{K-1} F_{k^{\prime}}^{(k)^{*}} R_{n, \bmod _{K M}\left(k K+k^{\prime}\right)} \quad k \in\{0,1, \ldots, M-1\}$

where the complex-valued coefficients $F_{k^{\prime}}^{(k)}$ in Equation 69 replace the coefficients $G_{k^{\prime}}$ in Equation 54; therefore, the coefficients $F_{k^{\prime}}^{(k)}$ can be set to $G_{k^{\prime}}$ obtaining the structure for a flat channel; on a multipath channel, we can set them in order to equalize the channel improving the receiver performance. The value of $\alpha$ to be used in Equation 43 is given by $\alpha \triangleq \sqrt{P_{r} / P_{s}}$ where $P_{r} \triangleq\left\langle E\left[|h[i] \otimes s[i]|^{2}\right]\right\rangle$ represents the average power of the useful component of the received signal $r[i]$ and $P_{s} \triangleq\left\langle E\left[|s[i]|^{2}\right]\right\rangle$ represents the average power of the transmitted signal $s[i]$.

The decision variable $D_{n, k}^{(R)}$ in Equation 69 can be written as follows:

$$
D_{n, k}^{(R)}=I_{0,0}^{(\mathrm{R}, k)} a_{n, k}^{R}+I_{n, k}+\eta_{n, k}
$$

where $I_{0,0}^{(\mathrm{R}, k)}$ represents the coefficient of the useful term $a_{n, k}^{R}, \eta_{n, k}$ describes the effect of the background noise, and $I_{n, k}$ describes the intersymbol and intercarrier interferences of the symbols $a_{n-m, k-q}^{R}((m, q) \neq(0,0))$ and $a_{n-m, k-q}^{I}$ on the useful symbol $a_{n, k}^{R}$. Such interferences would be negligible on a flat channel but they become significant on the multipath channel (see Equation 67).

It is easy to prove that $I_{n, k}$ can be written as a linear combination of the interfering symbols; the coefficients of such a combination can be written as

$$
\begin{aligned}
I_{m, q}^{(\mathrm{R}, k)} & =\Re\left\{(-1)^{m}\left(\mathbf{v}_{2 m, q}^{(k)^{H}} \mathbf{f}_{k}\right)^{*}\right\} \\
I_{m, q}^{(\mathrm{I}, k)} & =\Re\left\{(-1)^{m}\left(\mathbf{v}_{\left.2 m-1, q^{(}\right)^{H}} \mathbf{f}_{k}\right)^{*}\right\}
\end{aligned}
$$

where $I_{m, q}^{(\mathrm{R}, k)}$ and $I_{m, q}^{(\mathrm{I}, k)}$ are the coefficients of the symbols of $a_{n-m, k-q}^{R}$ and $a_{n-m, k-q}^{I}$, respectively. This shows that the coefficients $F_{k^{\prime}}^{(k)^{*}}$ of the receiver structure, collected in the $(2 K-1) \times 1$ vector

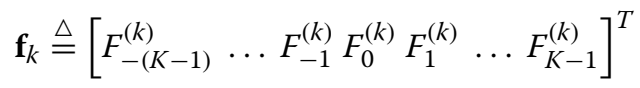

influence the useful coefficient and the interference power; we can, therefore, set the vector $\mathbf{f}_{k}$ in order to equalize the effects of the multipath channel in Equation 67.

The noise term $\eta_{n, k}$ in Equation 70 is a zero-mean complex-valued Gaussian random variable with variance $\frac{2\left\|\mathbf{f}_{k}\right\|^{2} \sigma_{a}^{2}}{\gamma\left\|\mathbf{G}^{(K)}\right\|^{2}}$ where $\gamma$ is defined as the signal-to-noise ratio per subcarrier, i.e., $\gamma \triangleq \frac{E_{s}}{N_{0}}$ where $E_{s}$ is defined as the energy of the useful term of the received signal in a multicarrier symbol period that is dedicated to each active subcarrier.

On a flat channel $(h[n]=\delta[n])$, we denote with $\mathbf{v}_{n, q}^{(\mathrm{FLAT}, k)}$ the vector $\mathbf{v}_{n, q}^{(k)}$ in Equation 71 ; its $k^{\prime}$ th component $v_{n, q, k^{\prime}}^{(\mathrm{FLAT}, k)}$ $\left(k^{\prime} \in\{(-K-1), \ldots,-1,0,1, \ldots, K-1\}\right)$ can be written as $v_{n, q, k^{\prime}}^{(\mathrm{ELAT}, k)}=\frac{1}{\left\|\mathbf{G}^{(K)}\right\|^{2}} j^{-n-q}(-1)^{k n} e^{j \pi \frac{k^{\prime}}{K}} \sum_{i=n M / 2}^{K M+n M / 2-1} g[i] e^{-j 2 \pi \frac{q K+k^{\prime}}{K M} i}$

Then, the properties of the prototype filter and the choice $\mathbf{f}_{k}=\mathbf{G}^{(K)}$ for setting the receiver coefficients guarantee that the useful coefficient $I_{0,0}^{(\mathrm{R}, k)} \simeq 1$ and the interference coefficients are practically null; in practice, the achieved signal-to-interference-ratio is around $65 \mathrm{~dB}$, i.e.,

$\mathbf{v}_{0,0}^{(\mathrm{FLAT}, k)^{H}} \mathbf{G}^{(K)} \simeq 1 \quad\left|\Re\left\{\mathbf{v}_{m, q}^{(\mathrm{FLAT}, k)^{H}} \mathbf{G}^{(K)}\right\}\right| \ll 1 \quad \forall(m, q) \neq(0,0)$

The satisfaction of the condition in Equation 74 is equivalent to the condition in Equation 44 and it concerns the design of the prototype filter. Moreover, it guarantees the optimality (in the maximum-likelihood sense) of the receiver structure on the flat channel.

When all the components of the vector $\mathbf{v}_{m, q}^{(F L A T, k)}$ are nonnull, the vector $\mathbf{v}_{m, q}^{(k)}$ can be written as

$$
\mathbf{v}_{m, q}^{(k)}=\mathbf{v}_{m, q}^{(\mathrm{FLAT}, k)} \times \mathbf{w}_{m, q}^{(k)}
$$

where the $k^{\prime}$ th component $w_{m, q, k^{\prime}}^{(k)}$ of the vector $\mathbf{w}_{m, q}^{(k)}$ is defined as

$$
w_{m, q, k^{\prime}}^{(k)}=\sum_{\ell=0}^{L_{h}} h[\ell] \frac{\sum_{i=m M / 2-\ell}^{K M+m M / 2-\ell-1} g[i] e^{-j 2 \pi \frac{q K+k^{\prime}}{K M} i}}{\sum_{i=m M / 2}^{K M+m M / 2-1} g[i] e^{-j 2 \pi \frac{q K+k^{\prime}}{K M} i}} e^{-j 2 \pi \frac{k K+k^{\prime}}{K M} \ell}
$$

When $v_{n, q, k^{\prime}}^{(\mathrm{FLAT}, k)}=0$, then the denominator of Equation 76 is null and Equation 75 is still valid provided that we replace with unit both the denominator of Equation 76 and the same quantity in Equation 73.

From Equations 71, 74, and 75, it follows that the choice

$$
\mathbf{f}_{k}=\mathbf{G}^{(K)} / \mathbf{w}_{m, q}^{(k) *}
$$

where we denote with / the component-wise division of the two vectors and $(m, q) \neq(0,0)$, implies ${ }^{\mathrm{c}}$ that

$$
\left|\Re\left\{\mathbf{v}_{m, q}^{(k)} \mathbf{f}_{k}\right\}\right| \ll 1
$$

However, a single vector $\mathbf{f}_{k}$ has to be chosen and, consequently, Equation 78 can be satisfied for a single value of $(m, q)$. A robust choice usually done for setting $\mathbf{f}_{k}$ lies in 
using Equation 77 with $(m, q)=(0,0)$, which implies that the useful coefficient is practically set to unit:

$$
\Re\left\{\mathbf{v}_{0,0}^{(k)^{H}} \mathbf{f}_{k}\right\} \simeq 1
$$

Such a derivation shows that also the optimum choice of $\mathbf{f}_{k}$ may not able to achieve the optimum performance achieved on a flat channel, unless the vector $\mathbf{w}_{m, q}^{(k)}$ exhibits a weak dependence on $(m, q)$ so that a single vector $\mathbf{f}_{k}$ can approximately satisfy the condition in Equation 77 for any value of $(m, q)$ or at least for the most important values of $(m, q)$. Such a weak dependence is present when $L_{h} \ll M$; in such a case, the quantity in Equation 76 can be approximated as follows:

$$
\frac{\sum_{i=m M / 2-\ell}^{K M+m M / 2-\ell-1} g[i] e^{-j 2 \pi \frac{q K+k^{\prime}}{K M} i}}{\sum_{i=m M / 2}^{K M+m M / 2-1} g[i] e^{-j 2 \pi \frac{q K+k^{\prime}}{K M} i}} \simeq 1 \quad \forall \ell \leq L_{h}
$$

and, consequently, the vector $\mathbf{w}_{m, q}^{(k)}$ can be approximated by the following vector $\mathbf{h}$, which is independent of $(m, q)$ :

$\mathbf{h}_{k} \triangleq\left[\begin{array}{lllll}H_{k K-(K-1)} & \ldots & H_{k K-1} H_{k K} H_{k K+1} \ldots H_{k K+K-1}\end{array}\right]^{T}$.

where $H_{m}$ denotes the multipath channel frequency response at the frequency $F=m /(K M)$. Consequently, the choice in Equation 77 with the use of the expression $\mathbf{h}_{k}$ for $\mathbf{w}_{m, q}^{(k)}$, i.e.,

$$
\mathbf{f}_{k}=\mathbf{G}^{(K)} / \mathbf{h}_{k}^{*} \Leftrightarrow F_{k^{\prime}}^{(k)}=\frac{G_{k^{\prime}}}{H_{k K+k^{\prime}}^{*}} .
$$

guarantees to the structure the same performance achieved on flat channel provided that $L_{h} \ll M$ (see Figure 1 for a scheme of the FS structure in Equation 69 when Equation 82 is chosen).

\subsection{Recalling the standard approach}

The standard approach consists in including an equalizer stage in cascade with the structure described in Subsection 3.1. The effects of the multipath channel can be equalized by using a single-tap structure [7]. In this case, the $k$ th entry of the DFT output (see Equations 50 or 51) is multiplied by $1 / H\left(F_{k}\right)$ with $F_{k}=\frac{k}{M}$ in the standard structure for $k \in\{0,1, \ldots, M-1\}$.

More sophisticated multitap structures could be used and have also been proposed with reference to the standard structure [22]. Since they operate in the frequency domain and at twice the multicarrier symbol rate, they introduce an additional delay proportional to the number of taps. We consider the single-tap equalizer in both structures since it maintains limited the overall latency of the transceiver. It may appear that the FS equalizer be equivalent to a multitap sub-channel equalizer following the standard structure and therefore that the considered comparison be unfair. However, they are not equivalent for two reasons: (a) because the delay introduced by the two structures is different and obtaining the minimum delay is important in a transceiver, like the OFDM/OQAM one, with an already larger delay in comparison with the OFDM system; (b) the PPN-FFT scheme performs equalization after sampling rate reduction which introduces an interpolation operation. The distinction of the two structures in terms of sampling rate reduction lies in the fact that the FS structure performs equalization in its internal behavior and therefore before sampling rate reduction while the PPN structure performs equalization after sampling rate reduction and consequently needs to use the single-tap equalizer to not increase the transceiver delay.

\subsection{Comparing the signal-to-interference-and-noise ratios of the two structures}

The equivalence of the two structures described in Section 3 on a flat channel implies that the behavior of the single-tap equalizer can be described with the same relations introduced in Subsection 4.1 provided that the coefficients expression in Equation 82 is replaced by the following one

$$
F_{k^{\prime}}^{(k)}=\frac{G_{k^{\prime}}}{H_{k K}^{*}} .
$$

In other terms, the choice in Equation 83 makes the FS receiver equivalent to the PPN structure equipped with the single-tap equalizer of coefficient $1 / H_{k K}$. Therefore, by comparing Equations 82 and 83, we can obviously note that the advantage of the frequency-despreading equalizer lies in its capability of using the coefficient $H_{k K+k^{\prime}}$ instead of the constant term $H_{k K}$. Since the FS structure in Equation 69 first extracts the DFT of the input vector $\mathbf{r}_{n}^{(K)}$ according to Equation 56 and subsequently uses the coefficient $F_{k^{\prime}}^{(k)}$ to equalize the channel effect at frequency $\left(k K+k^{\prime}\right) /(K M)=k / M+k^{\prime} /(K M)$, the FS structure with the choice in Equation 82 uses the right coefficient (i.e., $\left.H_{k K+k^{\prime}}\right)$ to equalize the channel response at frequency $\left(k K+k^{\prime}\right) /(K M)$ while the FS structure with the choice in Equation 83, which is equivalent to the PPN structure equipped with the single-tap equalizer, always uses the same coefficient $H_{k K}$ to equalize the channel responses at the different frequencies $\left(k K+k^{\prime}\right) /(K M)$ for $k^{\prime} \in$ $\{-(K-1), \ldots,-1,0,1, \ldots, K-1\}$. In other terms, differently from the PPN structure equipped with the single-tap equalizer, the FS structure is able to equalize with different coefficients the different parts of the subcarrier band. Since the effect of an offset $n_{\tau}$ in timing synchronization, perfectly compensated however in the frequency domain, 
can be obtained by setting $h[\ell]=\delta\left[\ell-n_{\tau}\right]$, our analysis shows that a performance improvement of the FS equalizer, which for $n_{\tau}=0$ (flat channel) is equivalent to the single-tap equalizer, appears when larger values of $n_{\tau}$ determine faster variations of the channel frequency response so that non-negligible variations appear within the subcarrier band; in such a case, the FS structure is able to use different coefficients to equalize each part of the subcarrier band and can therefore achieve improved performance in comparison with the PPN structure equipped with single-tap equalizer. Such a superior capability of the FS structure is irrelevant in the presence of a flat channel; therefore, the two structures show the same performance on the flat channel or on channels where the variations on the subcarrier band (of length $1 / M$ ) are marginal.

Furthermore, an unified expression of the signal-tointerference-and-noise ratio (SINR) on the $k$ th subcarrier, denoted as $\operatorname{SINR}_{k}$, of the two structures on the $k$ th subcarrier can be written as follows:

$$
\begin{aligned}
& \operatorname{SINR}_{k}= \\
& \frac{\mathfrak{R}^{2}\left\{\mathbf{v}_{0,0}^{(k)^{H}} \mathbf{f}_{k}\right\}}{\gamma\left\|\mathbf{G}^{(K)}\right\|^{2}}+\sum_{n=n_{\min }, n \neq 0}^{n_{\max }} \Re^{2}\left\{\mathbf{v}_{n, 0}^{(k) H} \mathbf{f}_{k}\right\}+\sum_{n=n_{\min }}^{n_{\max }} \sum_{q=1}^{M-1} \Re^{2}\left\{\mathbf{v}_{n, q}^{(k)^{H}} \mathbf{f}_{k}\right\}
\end{aligned}
$$

where the SINR of the frequency-despreading receiver is determined by the use of the vector $\mathbf{f}_{k}$ described in Equation 82 whereas the single-tap equalizer is determined by the use of the vector $\mathbf{f}_{k}$ in Equation 83. Note that the signal-to-interference ratio $\mathrm{SIR}_{k}$ can be obtained by employing Equation 84 without the first term at the denominator.

In order to plot the resulting SINRs for the two structures, we have to set the prototype filter $g[\cdot]$ : we set it to the filter designed in [5]. For the two structures, we report the SIR and the SINR for a choice of the subcarrier index $k=56, M=1024$, and by using 21 subcarriers and 21 time-interval values to approximate the sum in Equation 84. The channels employed to determine the receiver SINR are 1,000 random realizations of the ITU$R$ Vehicular B [23] and the SINR values, evaluated from Equation 84 and reported in Figures 2, 3, and 4, have been normalized to $\left|H_{k K}\right|^{2}$ in order to separate the effects due to the equalizer capability from that due to the fading of the $k$ th subcarrier.

Figure 5 shows that the frequency-despreading receiver is able to get the same SIR performance that it achieves on the flat channel on a large fraction of the considered different channels, randomly selected and ordered for decreasing values of output SIR achieved by the frequency-despreading receiver. The multipath channels where it cannot achieve the SIR achieved on the flat channel are those with very fast variations of the channel frequency response on the considered subcarrier. The single-tap shows poorer performance mainly because, on many channels realizations, the channel frequency response is not flat in the subcarrier band of size $1 / M$. From the SIR evaluation, it follows that, when we set the signal-to-noise ratio $\gamma$ between the SIR achieved by the

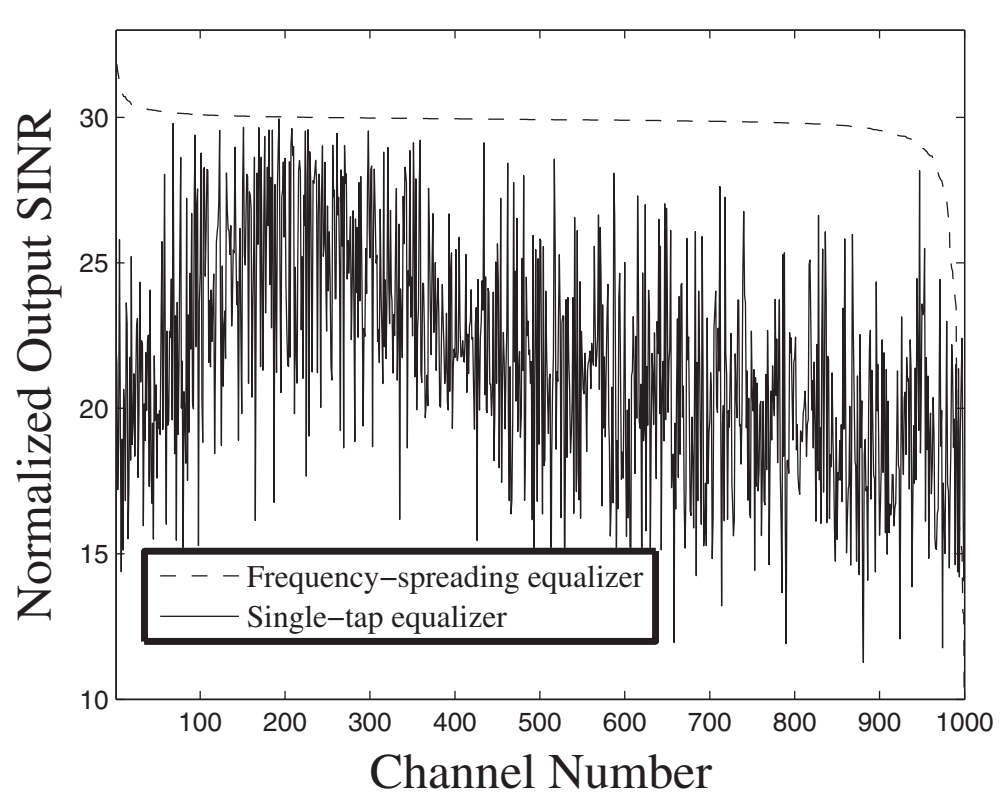

Figure 2 Samples of output SINR when $\gamma$ is $\mathbf{3 0} \mathbf{~ d B}$. The normalized output SINRs for the two structures on 1,000 different channel realizations according to the ITU-R Vehicular B model when $\gamma$ is $30 \mathrm{~dB}$. 


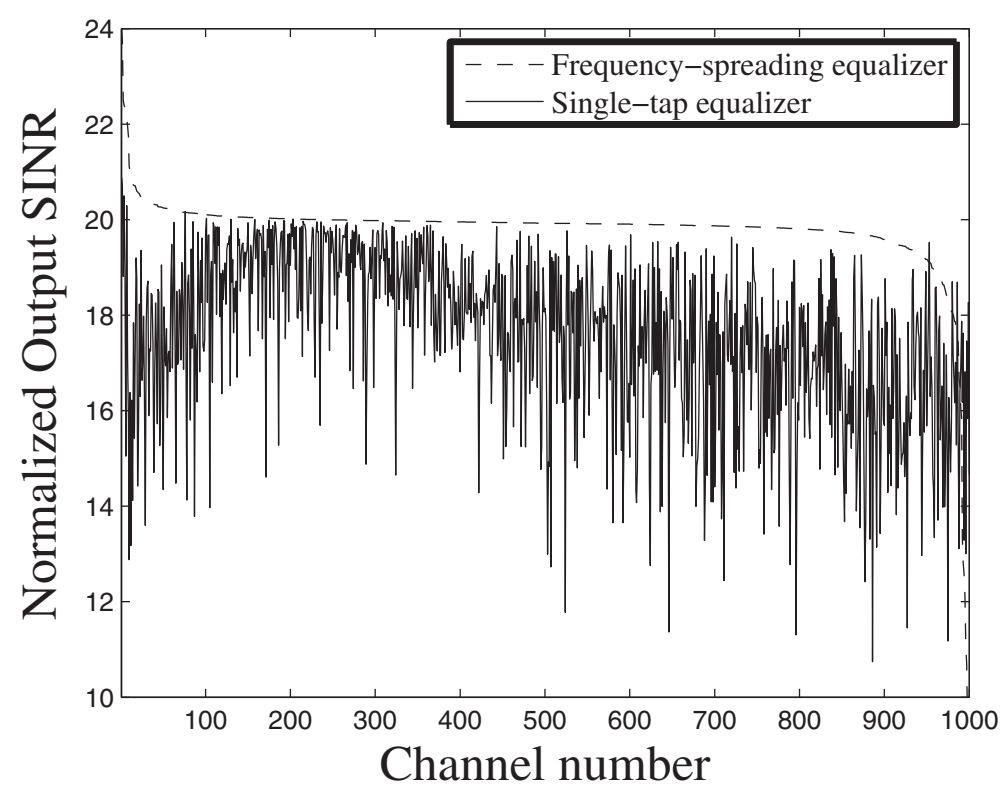

Figure 3 Samples of output SINR when $\gamma$ is $\mathbf{2 0} \mathbf{d B}$. The normalized output SINRs for the two structures on 1,000 different channel realizations according to the ITU-R Vehicular B model when $\gamma$ is $20 \mathrm{~dB}$.

single-tap equalizer and that achieved by the frequencyspreading one, the SIR limitation implies also a SINR limitation and, therefore, a performance advantage of the frequency-spreading structure is present. In fact, from Figures 2, 3, and 4, we can notice a significant advantage of the frequency-despreading equalizer when we set the value of $\gamma$ at $30 \mathrm{~dB}$; such advantage is reduced but it is still significant at $\gamma$ equal to $20 \mathrm{~dB}$ while the noise limitation becomes dominant for $\gamma$ equal to $10 \mathrm{~dB}$ and the frequency-despreading structure cannot take advantage from its superior interference-rejection capability.

\section{Performance comparison of the two structures}

In the present section, we assess via computer simulations the equalization performance achieved by using the frequency-spreading structure and compare it with

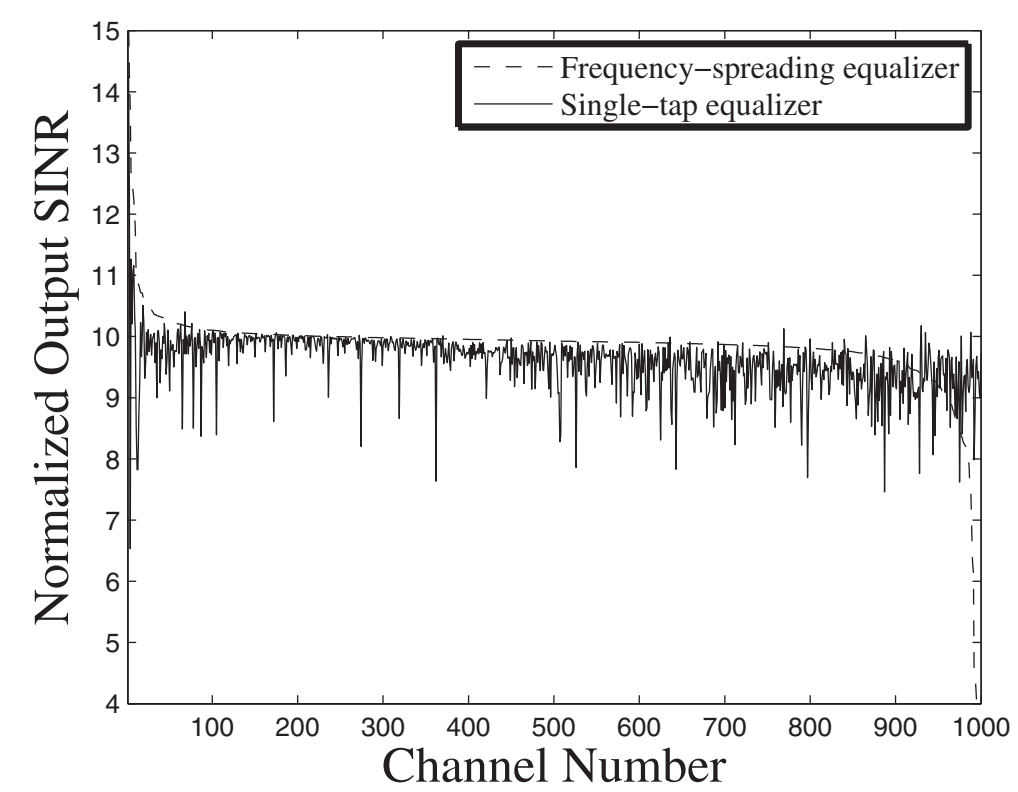

Figure 4 Samples of output SINR when $\gamma$ is $10 \mathrm{~dB}$. The normalized output SINRs for the two structures on 1,000 different channel realizations according to the ITU-R Vehicular B model when $\gamma$ is $10 \mathrm{~dB}$. 


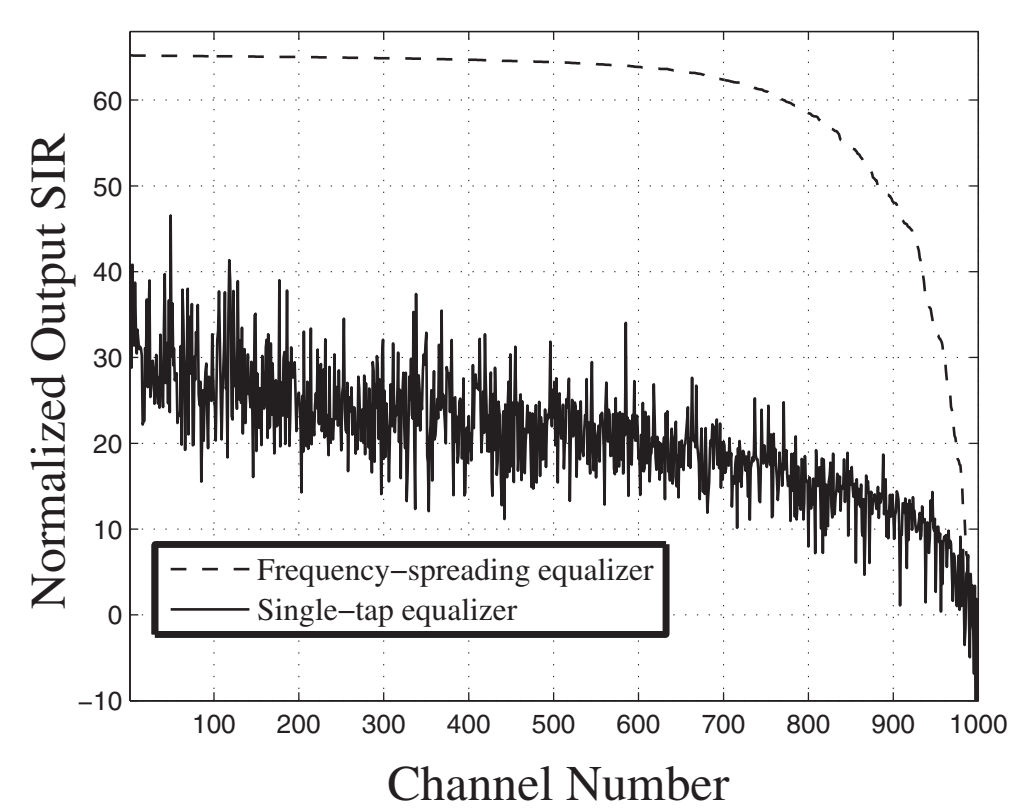

Figure 5 Samples of output SIR. Samples of the output SIRs for the two structures on 1,000 different channel realizations according to the ITU-R Vehicular B model.

that achieved by the standard structure. We have also included in the performance comparison the classical OFDM system that is often considered for opportunistic transmissions because it is a classical scheme employing the multicarrier approach where many practical difficulties have already been resolved; this has a strong impact on the overall cost. However, for the cognitive radio context, it lacks flexibility and it is likely to lead to poor spectral efficiency. Since the latency ${ }^{\mathrm{d}}$ of the FBMC receiver is $K$ times larger than that of the OFDM receiver with the same number of subcarriers, we have set the number of subcarriers in the OFDM transceiver $K$ times larger in order to compare two structures with the same latency. Moreover, with such a choice, the OFDM receiver and the FS-FBMC receiver perform the FFT procedure on the same size, though FS-FBMC has still to perform it to a rate $2 K$ times larger. In particular, we have used 2,048 subcarriers for OFDM while we have used only 512 subcarriers for FBMC transceiver and we have used $K \in\{2,3,4\}$ in order to verify the effect of the overlap factor.

A number of $10^{4}$ Monte Carlo trials has been performed under the following conditions:

1. The considered FBMC and OFDM systems have a bandwidth $\frac{1}{T_{\mathrm{s}}}=11.2 \mathrm{MHz}$

2. The transmitted symbols are the real and imaginary parts of 64-QAM symbols;

3. The considered multipath fading channel model is the ITU-R Vehicular B [23];
4. The used prototype filter is that proposed in [5]. Actually, any type of prototype filter can be implemented with an extended FFT, due to the equivalence between time and frequency domains. However, in order to be practical, the number of frequency domain filter coefficients must be the smallest possible, which is the case of the used filter;

5. The channel is fixed in each run but it is independent from one run to another;

6. The residual timing offset (RTO) and the normalized residual carrier frequency offset (RCFO) are controlled as simulation parameters;

7. Both systems exploit a one-tap subcarrier equalizer with perfect knowledge of the channel and of the residual timing error, i.e., when simulating the presence of the timing offset $n_{\tau}$, we have used $\exp \left(-j 2 \pi n_{\tau} k / M\right) / H(k / M)$ instead of $1 / H(k / M)$ as coefficient of the single-tap equalizer in the standard structure and $\exp \left(-j 2 \pi n_{\tau} k / K M\right) / H(k / K M)$ as coefficient of the single-tap equalizer for the frequency-despreading structure;

8. The effect of the RCFO on the phase of each decision variable in the frequency domain, which increases [20] linearly with time, is not compensated; therefore, the BER is dependent on the specific multicarrier symbol interval considered for equalization. In order to maintain sufficiently limited the effects of such nonideal receiver behavior, we evaluate the BER on the data transmitted in one of the first multicarrier symbol intervals, the eighth one; 
9. In order to use the same bandwidth in both FBMC and OFDM, which exhibits a larger spectral leakage, we have set the percentage of active subcarriers in OFDM transceiver as $82 \%$ of the overall number of subcarriers while we have set to $89 \%$ this percentage in OFDM/OQAM transceiver;

10. The length of the cyclic prefix is $1 / 8$ of the OFDM multicarrier symbol period (note that since in the FBMC system the cyclic prefix is not used, in the considered case an increase of the bit-rate nearly equal to $11.1 \%$ with respect to the OFDM system is obtained).

Note that, in consequence of the choices reported at the points 9 and 10, the data rate of the OFDM system is about $82 \%$ of the data rate of the FBMC system.

We first consider a simulation scenario where no timing or frequency offset is introduced; Figure 7 reports the results of the experiment. We can verify that the two FBMC structures, which are equivalent in the flat channel, perform differently on multipath channel because of the difference between the equalization mechanisms; more specifically, the frequency-spreading structure provides improved performance with respect to the standard structure and this improvement increases as the overlapping factor increases. Moreover, both structures exhibit a performance floor at large values of $E_{b} / N_{0}$. However, while the performance achieved by the standard FBMC receiver is very poor, in the range $0 \mathrm{~dB} \leq E_{b} / N_{0} \leq 20 \mathrm{~dB}$, the performance of the FS-FBMC system is practically equivalent to that of the OFDM transceiver employing 2,048 subcarriers for a sufficiently large value of the overlap parameter $K$. Since the channel coherence time may impose a smaller number of subcarriers also to the OFDM transceiver, it is interesting to test the OFDM system with only 512 subcarriers; the results not reported for clarity in Figure 7 show a poor performance, practically equivalent to the standard structure, since it is unable to equalize the considered channel (the length of the cyclic prefix $(512 / 8) T_{s}$ is smaller than the ITU-R Vehicular B channel length).

We have also performed other simulation experiments to verify the performance on the less hostile ITU-R Vehicular A channel: here, the condition $L_{h} \ll M$ is better satisfied. In fact, the corresponding results, shown in Figure 6, report that the two structures and the OFDM system are practically equivalent on channel A. Only for larger values of $E_{b} / N_{0}$ we can observe some difference; in particular, we note that the single-tap equalizer for $K=2$ provides the worst performance; moreover, the three dashed-line curves, corresponding to the single-tap equalizer for $K=3$ and $K=4$ as well as the frequency spreading structure for $K=2$. Only for $K=3$ and $K=4$ the frequency despreading structure behaves practically equivalent to the OFDM system. Therefore, we can conclude that similar performance is achieved by OFDM and FS-FBMC transceivers also if the latter uses only 512 subcarriers while the former uses 2,048 subcarriers.

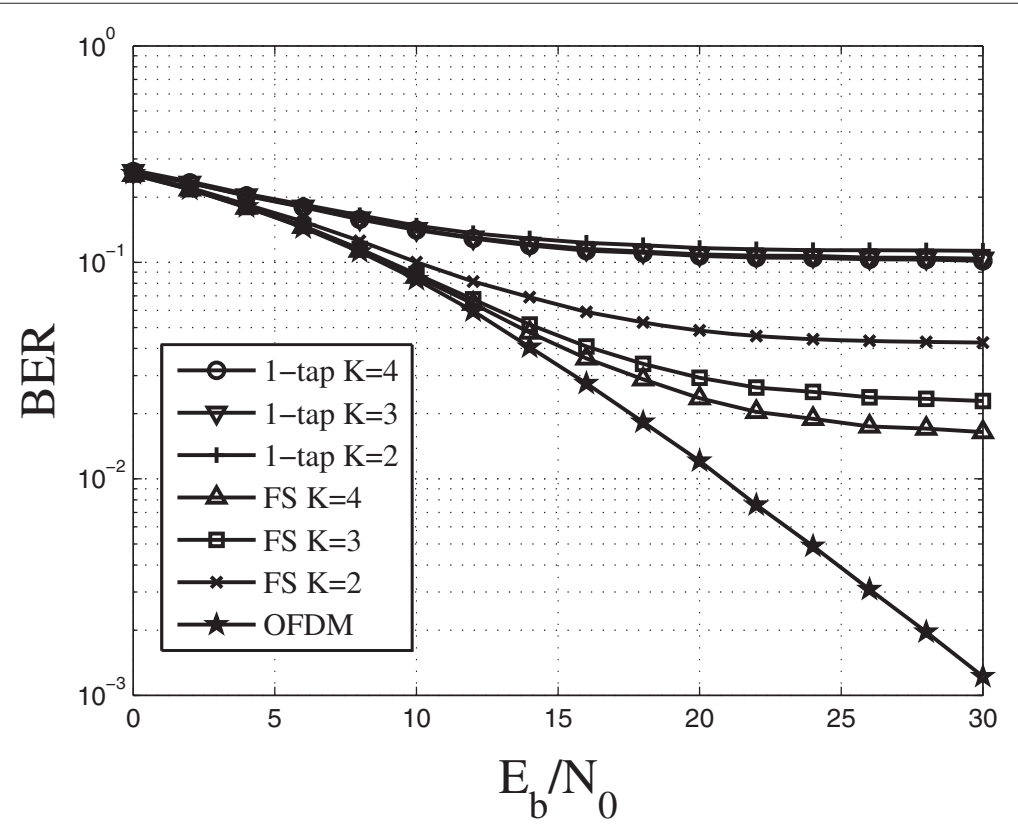

Figure 6 Comparison wrt $E_{b} / N_{0}$ (ITU-R Vehicular B channel). The effect of the noise on the performance of the OFDM transceiver (with 2,048 subcarriers) and of the two structures for FBMC transceiver (with 512 subcarriers) on ITU-R Vehicular B channel. Three possible values of the overlap parameters are considered. 


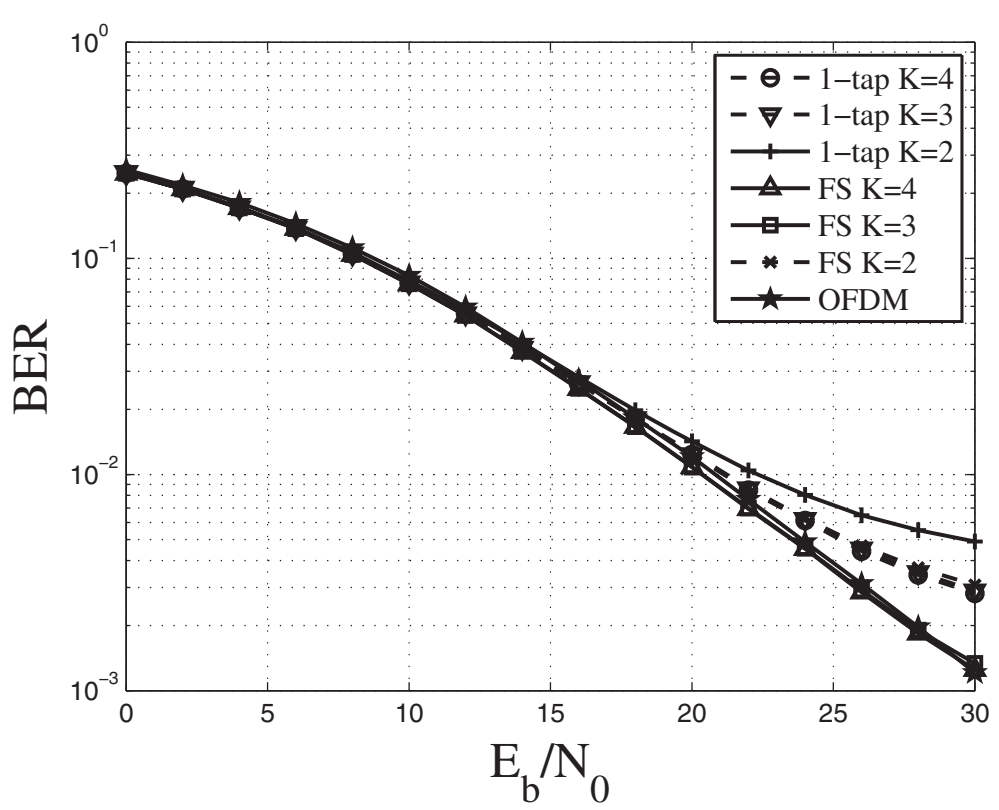

Figure 7 Comparison wrt $E_{b} / N_{0}$ (ITU-R Vehicular A channel). The effect of the noise on the performance of the OFDM transceiver (with 2,048 subcarriers) and of the two structures for FBMC transceiver (with 512 subcarriers) on ITU-R Vehicular A channel. Three possible values of the overlap parameters are considered.

In the second experiment, where we have imposed the value of the RTO, we have not introduced frequency offset and we have set $E_{b} / N_{0}$ equal to $20 \mathrm{~dB}$. The results are reported in Figure 8 and show the superior performance of the proposed FS-FBMC system. The presence of the cyclic prefix explains the threshold effect on the performance of the OFDM receiver; apart from this effect, we can verify that the OFDM performance worsens and becomes poor for larger values of the magnitude of the timing offset. The performance of the standard FBMC

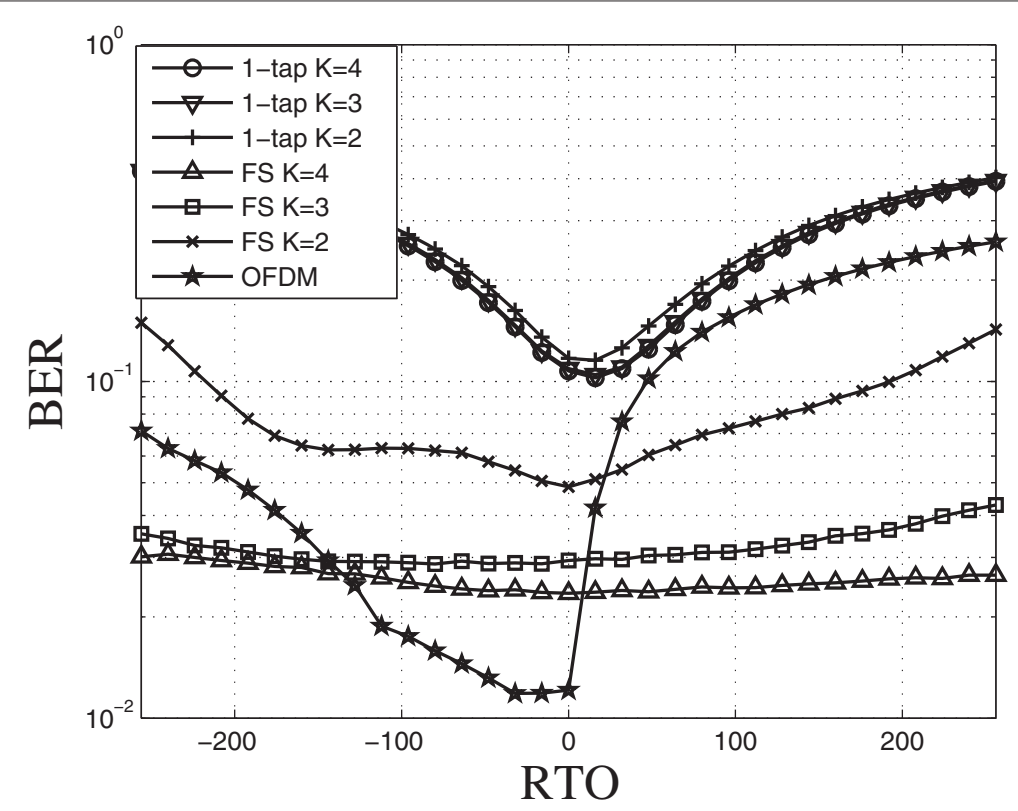

Figure 8 Comparison wrt RTO. The effect of a timing offset on the performance of the OFDM transceiver (with 2,048 subcarriers) and of the two considered structures for FBMC transceiver (with 512 subcarriers) on ITU-R Vehicular B channel. Three possible values of the overlap parameters are considered. 
structure is very poor independently of the value of the overlap parameter $K$ also in the absence of the timing offset and saturates for larger values of the magnitude of the RTO. Regarding the sensitivity of the performance to residual timing offset, it increases as the overlap parameter $K$ decreases.

Furthermore, in the third experiment, we have imposed the value of the RCFO, we have not introduced timing offset, and we have set again $E_{b} / N_{0}$ equal to $20 \mathrm{~dB}$. The results reported in Figure 9 show that the performance degradation of the FS-FBMC structure due to the RCFO is more pronounced for higher values of the overlap parameter $K$; however, such a degradation is lower than that of OFDM system. Moreover, when the CFO is larger than $5 / 100$, the performance of the OFDM and of the FS-FBMC structures is equivalent.

In order to better simulate the working conditions of a cognitive radio scenario, we next consider an uplink scenario where all active subcarriers are block-wise shared among four users whose delays are mutually independent and uniformly distributed within $\{-M / 2,-M / 2+$ $1, \ldots, M / 2-1\}$. Only a single subcarrier is left as guard between adjacent sets of active subcarriers whereas a maximum value of the normalized CFO equal to 0.1 is admitted on each user. The value of $K$ is set to 4 as, in the previous experiments, it resulted to be the best choice for $K$.

The considered uplink scenario is quite general and can be encountered in a number of cognitive network architectures, in particular when the cognitive cellular networks are taken into account [24]. For example, consider the case where the four terminals are located in an area where a large number of cells have been deployed by different operators for the local coverage enhancement of a potentially crowded zone interested in opportunistic multimedia downloads at low cost. Each relay is provided of a proper backhaul connection and cooperates with the other relays (and with the terminals) by means of a control channel, designed in dedicated, common, or underlay fashion [25]. The cooperation is aimed at determining according to an optimization procedure (e.g., that proposed in [26]), the subcarriers available for the transmission of each active terminal, and its transmitted power (to be minimized). Such a cooperation obviously does not include a timing alignment procedure not only because it would make much more complex the access control but mainly because it is impossible, i.e., the distances among relays imply that the transmission of each terminal is received by different relays. Therefore, in absence of a timing alignment procedure, the delay and the CFO of the user of interest are perfectly compensated and, then, all the other users remain asynchronous; moreover, we first consider the case where each signal, coming from the other users, at the receiver arrives with the same power of the useful signal.

The results, reported in Figure 10, show the BER versus $E_{b} / N_{0}$ for the user of interest. It is important to note that the OFDM system performance is equivalent on the

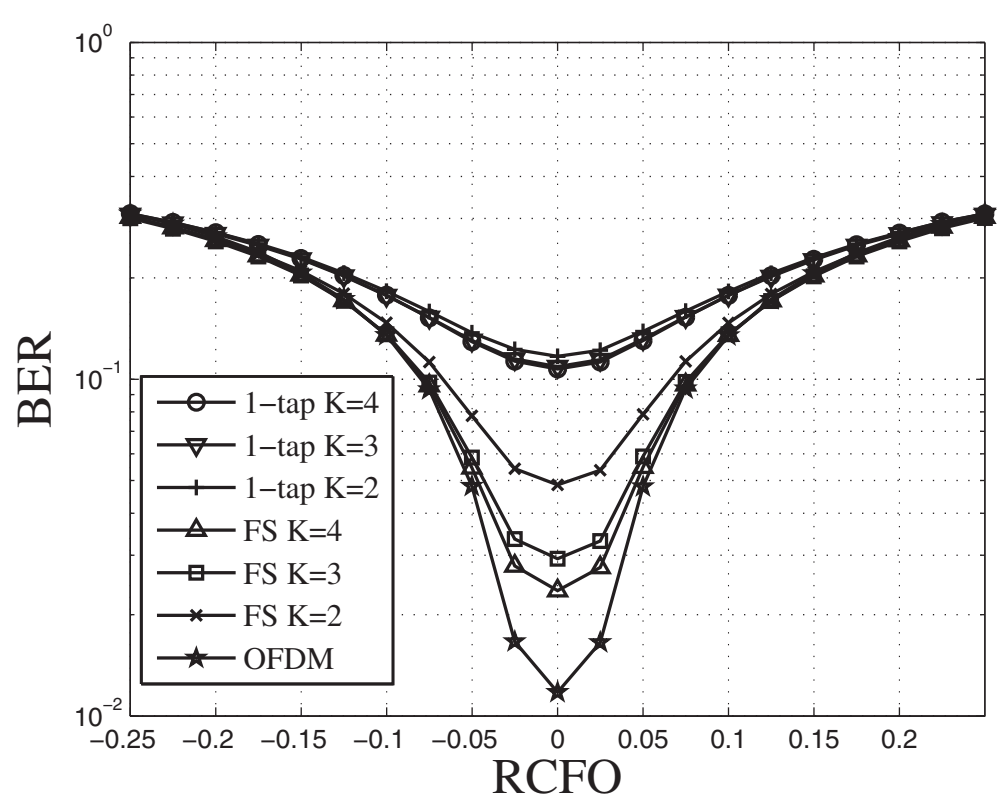

Figure 9 Comparison wrt CFO. The effect of a carrier offset on the performance of the OFDM transceiver (with 2,048 subcarriers) and of the two considered structures for FBMC transceiver (with 512 subcarriers) on ITU-R Vehicular B channel. Three possible values of the overlap parameters are considered. 


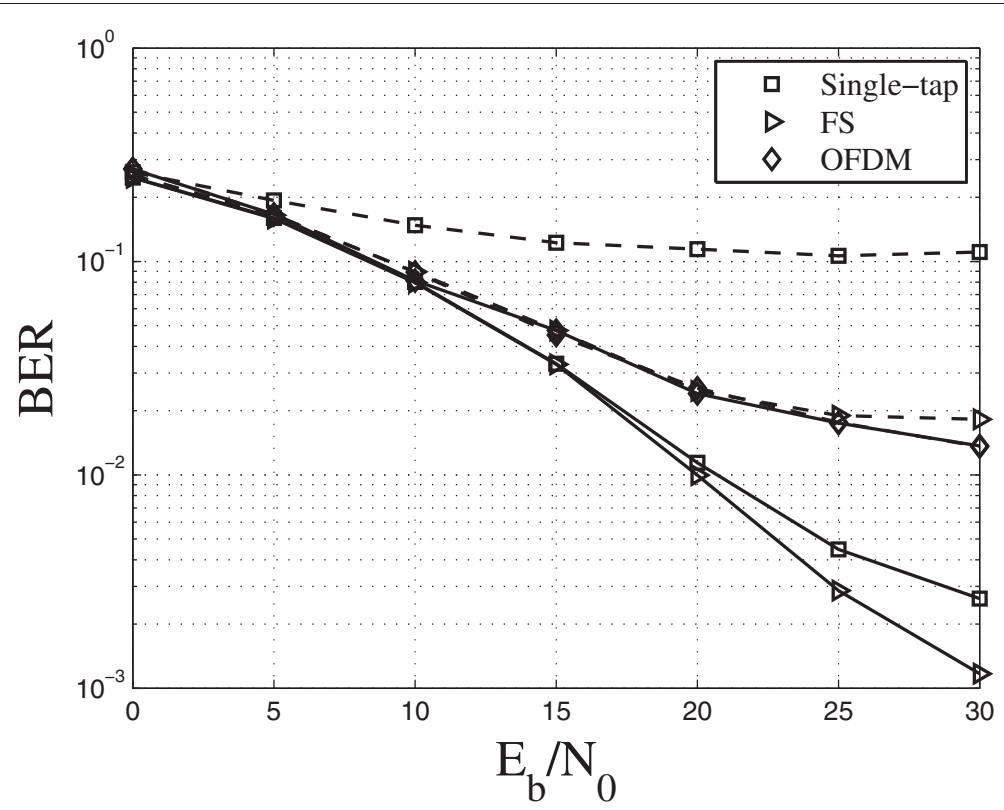

Figure 10 Comparison wrt $E_{b} / N_{0}$ (powers equal to that of the user of interest). The effect of the noise on the performance of the OFDM transceiver (with 2,048 subcarriers) and of the two structures for FBMC transceiver (with 512 subcarriers) on both the considered channels for $K=4$ in an uplink scenario with the powers of the interfering users equal to that of the user of interest. The continuous line refers to channel ITU-R Vehicular A while the dashed line refers to the ITU-R Vehicular B channel.

two channels and practically equivalent to the result of the FS system on channel ITU-R Vehicular B. The FS receiver performance is similar to that of the single-tap receiver on channel ITU-R Vehicular A and they achieve practically the same results achieved on the ideal flat channel. The single-tap receiver achieves very poor performance on channel ITU-R Vehicular B in comparison with the proposed frequency-despreading structure. Therefore, it is interesting to note that the FBMC system can provide comparable performance with the OFDM system only thanks to the receiver structure here proposed. We have also repeated the experiment when all the signals transmitted by interfering users arrive at the receiver with a power ten times larger than that of the useful signal; we have reported in Figure 11 the corresponding results. We note that the performance of FS structure is practically not affected by the larger powers of the interfering users whereas the OFDM system is severely affected and it exhibits a performance gap with respect to the FBMC system equipped with the frequency despreading receiver structure.

It is interesting to note that, differently from what happens on channel ITU-R Vehicular A, on channel ITU-R Vehicular $B$ the performance of the frequency despreading receiver is not equivalent to its performance on the flat channel. This is due to the fact that the powers of the interference terms are increased since the conditions that guarantee the optimality of the frequency despreading structure are not satisfied. Therefore, the interferences limit the performance for larger values of $E_{b} / N_{0}$. When the performance achieved by the frequency despreading structure is not satisfactory, transceiver performance can be improved by using another procedure for the design the coefficients $F_{k^{\prime}}^{(k)}$, more sophisticated than that in Equation 82. An alternative approach would require to introduce a time-domain filtering of each component of the DFT output, which would however also increase the receiver latency (however, the introduced latency would not be worse than that required by a lighter time-domain filtering approach following the PPN structure).

\section{Conclusions}

We have considered the FS-FBMC multicarrier scheme to meet requirements of cognitive radio such as high level of adjacent channel leakage and asynchronous communications. This structure is based on an FFT whose size is the length of the prototype filter and, then, the data samples are spread over several carriers. It has been shown that the FS-FBMC scheme is equivalent to the standard FBMC scheme in terms of input-output relation when operating on the flat channel and computationally more complex. However, it offers a crucial advantage in multipath channel where the FS structure can perform equalization in the frequency domain. The benefit of improved equalization with no additional delay for the user might well overweight the increase in complexity. In fact, the 


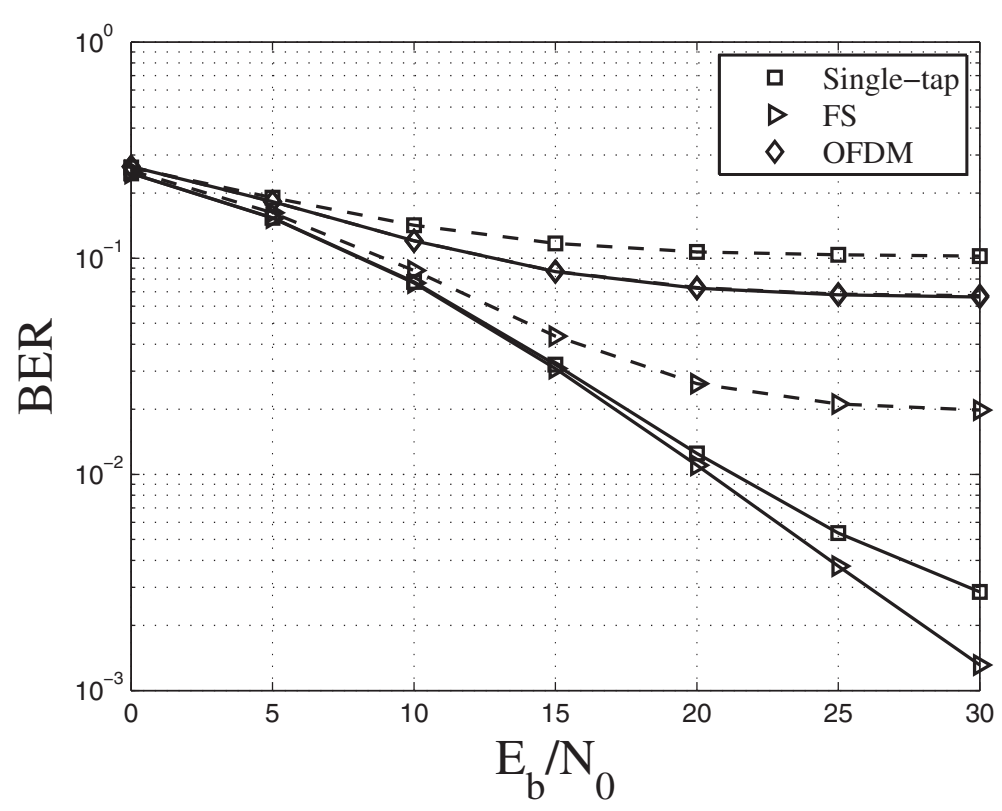

Figure 11 Comparison wrt $E_{b} / N_{0}$ (powers ten times larger than that of the user of interest). The effect of the noise on the performance of the OFDM transceiver (with 2,048 subcarriers) and of the two structures for FBMC transceiver (with 512 subcarriers) on both the considered channels for $K=4$ in an uplink scenario with the powers of the interfering users ten times larger than that of the user of interest. The continuous line refers to channel ITU-R Vehicular A while the dashed line refers to the ITU-R Vehicular B channel.

simulation results clearly show that the standard structure is not able to achieve satisfactory performance without using a multitap equalizer and, therefore, is not able, in multipath channel, to achieve the minimum transceiver latency achieved by the FS structure. Moreover, the equivalence proven in the paper shows that the increased complexity may be needed in the receiver only. Furthermore, it has been shown that the FS-FBMC transceiver can achieve, in a wide range of values of $E_{b} / N_{0}$, a performance equivalent to that of an OFDM system with the same FFT size and similar latency. Therefore, in a cognitive radio transmission context, the considered FS-FBMC system can assure the following advantages in comparison with an OFDM transceiver:

- smaller guard bands in the frequency domain, which means improved performance of the multiplexing scheme. Such a performance advantage can be quantified in terms of the overall bit-rate resulting from the use of OFDM/OQAM or equivalently can be quantified by comparing the complexity of the two transceivers since OFDM may need a heavy digital filtering of the signal to be transmitted in order to maintain similar overall bit-rate, as pointed out in [2];

- robustness to residual timing and carrier frequency offsets;

- capability to fully exploit fragmented spectrum;

- more flexibility in multiuser exploitation since asynchronous users can be accommodated.
The main disadvantage of the FS-FBMC receiver is related to the higher rate at which the FFT has to be performed when $M / K$ subcarriers are employed in the frequency-spreading structure in comparison with the classical OFDM transceiver employing $M$ carriers. Here, however, a pipelining approach to the FFT implementation may significantly reduce the importance of such a disadvantage.

\section{Endnotes}

${ }^{\mathrm{a}}$ The adjacent channel leakage ratio (ACLR) is the ratio of the filtered mean power centered on the assigned channel frequency to the filtered mean power on the adjacent channel frequency.

${ }^{\mathrm{b}}$ We use the approximate expression $4 M \log (M)-$ $6 M+8$ as a first approximation and to better appreciate the behavior of the complexity; however, the exact calculation of the number of flops for IFFT is given [27] by $\frac{34}{9} M \log (M)-\frac{124}{27} M-2 \log (M)+\frac{16}{27}(-1)^{\log (M)}(1-$ $3 / 8 \log (M))+8$.

${ }^{\mathrm{c}}$ Also if a component of the vector $\mathbf{w}_{m, q}^{(k)}$ is null the condition existing on a flat channel can be still restored provided that we accept that the definition (77), which does not apply to such a case, is obviously meant in the sense that the corresponding component of the vector $\mathbf{f}_{k}$ is null.

${ }^{\mathrm{d}}$ The delay of each symbol transmitted with OFDM/OQAM system is equal to the length of the 
prototype filter while the delay of each symbol transmitted with OFDM system is equal to the length of the multicarrier symbol period. Thus, the FBMC and the OFDM transceivers have the same delay if the OFDM one has $K$ times more sub-carriers than OFDM/OQAM.

\section{Appendix A}

By using Equations 7, 8, and 21, the $k$ th component $d_{n, k}^{(R)}$ of $\mathbf{d}_{n}^{(R)}$ can be written as:

$$
\begin{aligned}
d_{n, k}^{(R)}= & \sum_{n^{\prime}=0}^{N_{b}+N_{s}-1} \sum_{k^{\prime} \in \mathcal{A}} c_{n^{\prime}, k^{\prime}}^{(R)} e^{j \frac{2 \pi}{M} k k^{\prime}} g\left[k+\left(n-n^{\prime}\right) M\right] \\
& k \in\{, 1, \ldots, M-1\} \\
= & \sum_{n^{\prime}=n-(K-1)}^{n} \sum_{k^{\prime} \in \mathcal{A}} c_{n^{\prime}, k^{\prime}}^{(R)} c^{j \frac{2 \pi}{M} k k^{\prime}} g\left[k+\left(n-k^{\prime}\right) M\right] \\
& +\sum_{k^{\prime} \in \mathcal{A}} c_{n-2, k^{\prime}}^{(R)} e^{j \frac{2 \pi}{M} k k^{\prime}} g[k]+\sum_{k^{\prime} \in \mathcal{A}} c_{n-1, k^{\prime}}^{(R)} e^{j \frac{2 \pi}{M}(k+M) k^{\prime}} g[k+M] \\
& +\sum_{k^{\prime} \in \mathcal{A}} c_{n-3, k^{\prime}}^{(R)} e^{j \frac{2 \pi}{M}(k+3 M) k^{\prime}} g[k+3 M]+\ldots \\
& +\sum_{k^{\prime} \in \mathcal{A}} c_{n-(K-1), k^{\prime}}^{(R)} e^{j \frac{2 \pi}{M}(k+(K-1) M) k^{\prime}} g[k+(K-1) M]
\end{aligned}
$$

Using Equation 20 in A.1, Equation 19 directly follows.

\section{Appendix B}

The $\ell$ th component $z_{n, \ell}$ of the vector $\mathbf{z}_{n}$ can be calculated by circular convolution of the two vectors $\mathbf{G}$ and $\mathbf{c}_{n}$

$$
\begin{aligned}
z_{n, \ell} & =\sum_{m=0}^{K M-1} c_{n, m}^{(K R)} G_{\bmod _{K M}(\ell-m)} \quad \ell \in\{0,1, \ldots, K M-1\} \\
& =\sum_{q=0}^{M-1} c_{n, q}^{(R)} G_{\bmod _{K M}(\ell-q K)}=\sum_{q \in \mathcal{A}} c_{n, q}^{(R)} G_{\bmod _{K M}(\ell-q K) .}
\end{aligned}
$$

Using Equation 33, $z_{n, \ell}$ in Equation B.1 for $\ell=p K$ and $p \in\{0,1, \ldots, M-1\}$ can be written as

$$
\begin{aligned}
z_{n, p K} & =\sum_{m=0}^{M-1} c_{n, m}^{(R)} G_{\bmod _{K M}(p K-m K)} \\
& =G_{0} \sum_{m=0}^{M-1} c_{n, m}^{(R)} \delta[p-m]=c_{n, p}^{(R)} G_{0} .
\end{aligned}
$$

Moreover, $z_{n, \ell}$ in Equation B.1 for $\ell=p K+k$ with $p \in$ $\{0,1, \ldots, M-1\}$ and $k \in\{1,2, \ldots, K-1\}$

$$
\begin{aligned}
z_{n, p K+k} & =\sum_{m=0}^{M-1} c_{n, m}^{(R)} G_{\bmod _{K M}(p K+k-m K)} \\
& =\sum_{m=0}^{M-1} c_{n, m}^{(R)}\left[G_{k} \delta[p-m]+G_{k-K} \delta\left[\bmod _{M}(p+1-m)\right]\right] \\
& =c_{n, p}^{(R)} G_{k}+c_{n, \bmod _{M}(p+1)}^{(R)} G_{k-K} .
\end{aligned}
$$

Consequently, taking into account Equations B.2, B.3, and 21 , for $p \in\{0,1, \ldots, M-1\}$ and $k \in\{0,1, \ldots, K-1\}$, we can compactly write

$$
\begin{aligned}
z_{n, p K+k} & =c_{n, p}^{(R)} G_{k}+c_{n, \bmod _{M}(p+1)}^{(R)} G_{k-K}(1-\delta[k]) \\
& =j^{p} a_{n, p}^{R} G_{k}+j^{\bmod _{M}(p+1)} a_{n, \bmod _{M}(p+1)}^{R} G_{k-K}(1-\delta[k]) \\
& =j^{p} a_{n, p}^{R} G_{k}+j^{p+1} a_{n, \bmod _{M}(p+1)}^{R} G_{k-K}(1-\delta[k])
\end{aligned}
$$

From Equation B.4, Equation 35 directly follows.

In the derivation, we have used the property that $j^{\bmod _{M}(p+1)}=j^{p+1}$ that holds for $p \in\{0,1, \ldots, M-1\}$ under the condition, always satisfied, that $M$ is multiple of 4. In fact, the property is trivial for $p \in\{0,1, \ldots, M-2\}$ since $\bmod _{M}(p+1)=p+1$ while, for $p=M-1$, it is equivalent to the property $j^{M}=1$, which holds only for $M$ integer multiple of 4 .

\section{Appendix C}

From Equations 42 and 14, it follows that

$$
\begin{aligned}
D_{n, k}^{(I)}= & \frac{-j}{A} j^{-k}\left\{\sum_{i=0}^{M-1} r[n M+M / 2+i] e^{-j k \frac{2 \pi}{M} i} g[i]\right. \\
& +\sum_{i=0}^{M-1} r[(n+1) M+M / 2+i] e^{-j k \frac{2 \pi}{M} i} g[M+i]+\ldots \\
& \left.+\sum_{i=0}^{M-1} r[(n+K-1) M+M / 2+i] e^{-j k \frac{2 \pi}{M} i} g[(K-1) M+i]\right\} .
\end{aligned}
$$

By using Equations 13 and 53, let us introduce the vector

$$
\mathbf{r}_{n+\ell}^{(I, g, n)} \triangleq \mathbf{r}_{n+\ell}^{(I)} \times \mathbf{g}_{\ell} \quad \ell \in\{0,1, \ldots, K-1\}
$$


and let us denote with $r_{n+\ell, i}^{(I, g, n)}$ its $i$ th component for $i \in\{0,1, \ldots, M-1\}$. Using such definitions, from Equation C.1, it follows that

$$
\begin{aligned}
A j^{k+1} D_{n, k}^{(I)} \triangleq & \sum_{q=0}^{M-1} r_{n, q}^{(I, g, n)} e^{-j k \frac{2 \pi}{M} q}+\sum_{q=0}^{M-1} r_{n+1, q}^{(I, g, n)} e^{-j k \frac{2 \pi}{M} q} \\
& +\sum_{q=0}^{M-1} r_{n+2, q}^{(I, g, n)} e^{-j k \frac{2 \pi}{M} q}+\ldots \\
& +\sum_{q=0}^{M-1} r_{n+(K-1), q}^{(I, g, n)} e^{-j k \frac{2 \pi}{M} q}
\end{aligned}
$$

From Equations 52 and C.3, it follows that

$$
\begin{aligned}
j A \mathbf{D}_{n}^{(I)} \times \mathbf{w}= & M \operatorname{DFT}\left[\mathbf{r}_{n}^{(I, g, n)}\right]+M \operatorname{DFT}\left[\mathbf{r}_{n+1}^{(I, g, n)}\right] \\
& +\ldots+M \operatorname{DFT}\left[\mathbf{r}_{n+(K-1)}^{(I, g, n)}\right] \\
= & M \sum_{\ell=0}^{K-1} \operatorname{DFT}\left[\mathbf{r}_{n+\ell}^{(I)} \times \mathbf{g}_{\ell}\right]
\end{aligned}
$$

The last equality can be equivalently re-written as in Equation 51.

\section{Appendix D}

From Equations 42 and 14, it follows that for $k \in\{0,1, \ldots$, $M-1\}$

$$
\begin{aligned}
D_{n, k}^{(I)} & =\frac{-j}{A} j^{-k} \sum_{i=0}^{K M-1} r[n M+M / 2+i] e^{-j k \frac{2 \pi}{M} i}\left\{\sum_{k^{\prime}=-(K-1)}^{K-1} G_{k^{\prime}} e^{j \frac{2 \pi}{K M} i k^{\prime}}\right\} \\
& =\frac{-j}{A} j^{-k} \sum_{k^{\prime}=-(K-1)}^{K-1} G_{k^{\prime}}^{*} \sum_{i=0}^{K M-1} r[n M+M / 2+i] e^{-j \frac{2 \pi}{K M} i\left(k K+k^{\prime}\right)}
\end{aligned}
$$

where we have taken into account that the prototype filter is real $\left(G_{-k}=G_{k}^{*}\right)$. Using Equations 63 and 64, Equation D.1 can be re-written as

$D_{n, k}^{(I)}=\frac{-j}{A} j^{-k} K M \sum_{k^{\prime}=-(K-1)}^{K-1} G_{k^{\prime}}^{*} R_{n, \bmod _{K M}\left(k K+k^{\prime}\right)}^{(I)} k \in\{0,1, \ldots, M-1\}$.

Taking into account Equations 62 and 59, Equation D.2 can be written as in Equation 61.

\section{Competing interests}

The authors declare that they have no competing interests.

\section{Author details}

${ }^{1}$ Dipartimento di Ingegneria Elettrica e delle Tecnologie dell'Informazione, Università degli Studi di Napoli Federico II, via Claudio 21, 80125 Napoli, Italy. ${ }^{2}$ CNAM-Electronique, 292 rue Saint-Martin, 75141, Paris cedex 03, France.

Received: 31 July 2014 Accepted: 31 December 2014

Published online: 10 March 2015

\section{References}

1. M Fitch, M Nekovee, S Kawade, K Briggs, R MacKenzie, Wireless service provision in TV white space with cognitive radio technology: a telecom operator's perspective and experience. IEEE Commun. Mag. 49(3), 64-73 (2011)

2. D Noguet, M Gautier, V Berg, Advances in opportunistic radio technologies for TWWS. EURASIP J. Wireless Commun. Netw. 2011(1), 170 (2011)

3. W Jiang, M Schellmann, in Proc. of IEEE Global Communications Conference (GLOBECOM 2012). Suppressing the out-of-band power radiation in multicarrier systems: a comparative study (Anahem CA, USA, 2012)

4. H Bogucka, A. M Wyglinski, S Pagadarai, A Kliks, Spectrally agile multicarrier waveforms for opportunistic wireless access. IEEE Commun. Mag. 49(6), 108-115 (2011)

5. M Bellanger, in Proc. of 2001 IEEE International Conference on Acoustics, Speech, and Signal Processing (ICASSP'01). Specification and design of a prototype filter for filter bank based multicarrier transmissions (Salt Lake City, UT, USA, 2001)

6. B Le Floch, M Alard, C Berrou, Coded orthogonal frequency division multiplex. Proc. IEEE. 83(6), 982-996 (1995)

7. B Farhang-Boroujeny, OFDM versus filter bank multicarrier. IEEE Signal Process. Mag. 28(3), 92-112 (2011)

8. KW Martin, Small sidelobe filter design for multitone data-communication applications. IEEE Trans. Circuits Syst.CAS-II. 45(8), 1155-1161 (1988)

9. M Bellanger, in Proc. of the 5th Symposium on Communications, Control and Signal Processing (ISCCSP 2012). FS-FBMC: an alternative scheme for filter bank based multicarrier transmission (Rome, Italy, 2012)

10. D Mattera, M Tanda, M Bellanger, in 2012 International Symposium on Wireless Communication Systems. Frequency-spreading implementation of OFDM/OQAM systems (Paris, France, 2012)

11. E Kofidis, A. A Rontogiannis, in 2010 IEEE 21st International Symposium on Personal Indoor and Mobile Radio Communications. Adaptive BLAST decision-feedback equalizer for MIMO-FBMC/OQAM systems (Istanbul, Turkey, 2010)

12. M Newinger, LG Baltar, AL Swindlehurst, JA Nossek, in 2010 IEEE 21st International Symposium on Personal Indoor and Mobile Radio Communications. MISO broadcasting FBMC system for highly frequency selective channels (Istanbul, Turkey, 2010)

13. T Ihalainen, A Ikhlef, J Louveaux, M Renfors, Channel equalization for multi-antenna FBMC/OQAM receivers. IEEE Trans. Vehicular Technol. 60(3), 2070-2085 (2011)

14. M Renfors, J Yli-Kaakinen, FJ Harris, Analysis and design of efficient and flexible fast-convolution based multirate filter banks. IEEE Trans. Signal Process. 62(15), 3768-3783 (2014)

15. M Renfors, J Yli-Kaakinen, in The Tenth International Symposium on Wireless Communication Systems 2013. Timing offset compensation in fast-convolution filter bank based waveform processing, (2013), pp. 1-4

16. P Siohan, C Siclet, N Lacaille, Analysis and design of OFDM/OQAM systems based on filterbank theory. IEEE Trans. Signal Process. 50(5), 1170-1183 (2002)

17. M Bellanger, J Daguet, TDM-FDM transmultiplexer: Digital polyphase and FFT. IEEE Trans. Commun. 22(9), 1199-1205 (1974)

18. B Hirosaki, An orthogonally multiplexed QAM system using the discrete Fourier transform. IEEE Trans. Commun. 29(7), 982-989 (1981)

19. H Sorensen, M Heideman, C Burrus, On computing the split-radix FFT. IEEE Trans. Acoust. Speech Signal Process. 34(1), 152-156 (1986)

20. H Saeedi-Sourck, Y Wu, JWM Bergmans, S Sadri, B Farhang-Boroujeny, Sensitivity analysis of offset QAM multicarrier systems to residual carrier frequency and timing offsets. Signal Process. 91(7), 1604-1612 (2011)

21. H Lin, M Gharba, P Siohan, Impact of time and carrier frequency offsets on the FBMC/OQAM modulation scheme. Signal Process. 102(9), 151-162 (2014)

22. T Ihalainen, TH Stitz, M Rinne, M Renfors, Channel equalization in filter bank based multicarrier modulation for wireless communications. EURASIP J. Adv. Signal Process. 2007(Article ID 49389), 18 (2007)

23. International Telecommunication Union, in Recommendation ITU-R M. 1225: Guidelines for evaluation of radio transmission technologies for IMT-2000, (ITU, 1997)

24. X Hong, J Wang, C-X Wang, J Shi, Cognitive radio in 5G: a perspective on energy-spectral efficiency trade-off. IEEE Commun. Mag. 52(7), 46-53 (2014) 
25. DL Wasden, H Moradi, B Farhang-Boroujeny, Design and implementation of an underlay control channel for cognitive radios. IEEE J. Select. Areas Commun. 30(10), 1875-1889 (2012)

26. M Shaat, F Bader, in Proceedings of the Fifth International Conference on Cognitive Radio Oriented Wireless Networks Communications (CROWNCOM). An uplink resource allocation algorithm for OFDM and FBMC based cognitive radio systems (Cannes, France, 2010)

27. FG Johnson, M Frigo, A modified split-radix FFT with fewer arithmetic operations. IEEE Trans. Signal Process. 55(1), 111-119 (2007)

\section{Submit your manuscript to a SpringerOpen ${ }^{\mathcal{O}}$ journal and benefit from:}

- Convenient online submission

- Rigorous peer review

- Immediate publication on acceptance

- Open access: articles freely available online

- High visibility within the field

- Retaining the copyright to your article

Submit your next manuscript at $\boldsymbol{\wedge}$ springeropen.com 